\title{
An efficient way to assemble finite element matrices in vector languages
}

\author{
François Cuvelier • Caroline Japhet • \\ Gilles Scarella
}

Received: date / Accepted: date

\begin{abstract}
Efficient Matlab codes in 2D and 3D have been proposed recently to assemble finite element matrices. In this paper we present simple, compact and efficient vectorized algorithms, which are variants of these codes, in arbitrary dimension, without the use of any lower level language. They can be easily implemented in many vector languages (e.g. Matlab, Octave, Python, Scilab, R, Julia, C++ with STL,...). The principle of these techniques is general, we present it for the assembly of several finite element matrices in arbitrary dimension, in the $\mathbb{P}_{1}$ finite element case. We also provide an extension of the algorithms to the case of a system of PDE's. Then we give an extension to piecewise polynomials of higher order. We compare numerically the performance of these algorithms in Matlab, Octave and Python, with that in FreeFEM++ and in a compiled language such as C. Examples show that, unlike what is commonly believed, the performance is not radically worse than that of $\mathrm{C}$ : in the best/worst cases, selected vector languages are respectively $2.3 / 3.5$ and 2.9/4.1 times slower than $\mathrm{C}$ in the scalar and vector cases. We also present numerical results which illustrate the computational costs of these algorithms compared to standard algorithms and to other recent ones.
\end{abstract}

Keywords finite elements, matrix assembly, vectorization, vector languages, Matlab, Octave, Python

Mathematics Subject Classification (2000) 65N30, 65Y20, 74S05

This work was partially funded by GNR MoMaS, CoCOA LEFE project, ANR DEDALES and MathSTIC (University Paris 13)

F. Cuvelier · G. Scarella

Université Paris 13, Sorbonne Paris Cité, LAGA, CNRS UMR 7539, 99 Avenue J-B Clément, F-93430 Villetaneuse, France, E-mail: cuvelier@math.univ-paris13.fr, scarella@math.univparis13.fr

C. Japhet

Université Paris 13, Sorbonne Paris Cité, LAGA, CNRS UMR 7539, 99 Avenue J-B Clément, F-93430 Villetaneuse, France. INRIA Paris-Rocquencourt, BP 105, F-78153 Le Chesnay, France, E-mail: japhet@math.univ-paris13.fr 


\section{Introduction}

Vector languages 1 such as Matlab [27, GNU Octave [30, Python [13, R 14, Scilab [12, Julia 22, C++ with STL,..., are very widely used for scientific computing (see for example $3,25,20,24,32$ ) and there is significant interest in programming techniques in these languages for two reasons. The first concerns how to make clear, compact code to ease implementation and understanding, which is important for teaching and rapid-prototyping in research and industry. The second concerns how to make this compact code fast enough for realistic simulations.

On the other hand, in finite element simulations [4, 5, 21, 33, 35, the need for efficient algorithms for assembling the matrices may be crucial, especially when the matrices may need to be assembled several times. This is the case for example when simulating time-dependent problems with explicit or implicit schemes with time-dependent coefficients (e.g. in ocean-atmosphere coupling or porous medium applications). Other examples are computations with a posteriori estimates when one needs to reassemble the matrix equation on a finer mesh, or in the context of eigenvalue problems where assembling the matrix may be costly. In any event, assembly remains a critical part of code optimization since solution of linear systems, which asymptotically dominates in large-scale computing, could be done with the linear solvers of the different vector languages.

In a vector language, the inclusion of loops is a critical performance degrading aspect and removing them is known as a vectorization. In finite element programming, the classical finite element assembly is based on a loop over the elements (see for example 26]). In 9] T. Davis describes different assembly techniques applied to random matrices of finite element type. A first vectorization technique is proposed in [9]. Other more efficient algorithms in Matlab have been proposed recently in $[1,2,3,8,15,17,23,34$.

In this paper we describe vectorized algorithms, which are variants of the codes in 2, 3, 15, 23, extended to arbitrary dimension $d \geq 1$, for assembling large sparse matrices in finite element computations. A particular strength of these algorithms is that they make using, reading and extending the codes easier while achieving performance close to that of $\mathrm{C}$.

The aim of this article is the quantitative studies for illustrating the efficiency of the vector languages and the various speed-up of the algorithms, relatively to each other, to $\mathrm{C}$ and to FreeFem++ [19. We also propose a vectorized algorithm in arbitrary dimension which is easily transposable to matrices arising from PDE's such as (see [33])

$$
-\nabla \cdot(\mathbb{A} \nabla u)+\nabla \cdot(\boldsymbol{b} u)+\boldsymbol{c} \cdot \nabla u+a_{0} u=f \quad \text { in } \Omega,
$$

where $\Omega$ is a bounded domain of $\mathbb{R}^{d}(d \geq 1), \mathbb{A} \in\left(L^{\infty}(\Omega)\right)^{d \times d}, \boldsymbol{b} \in\left(L^{\infty}(\Omega)\right)^{d}$, $c \in\left(L^{\infty}(\Omega)\right)^{d}, a_{0} \in L^{\infty}(\Omega)$ and $f \in L^{2}(\Omega)$ are given functions. The description of the vectorized algorithm is done in three steps: we recall (non-

\footnotetext{
1 which contain usual element-wise operators and functions on multidimensional arrays
} 
vectorized) versions called base and OptV1. The latter requires sparse matrix tools found in most of the languages used for computational science and engineering. Then we give vectorized algorithms which are much faster: OptV2 (memory consuming), OptV (less memory consuming) and OptVS (a symmetrized version of $\mathrm{OptV}$ ). These algorithms have been tested for several matrices (e.g. weighted mass, stiffness and elastic stiffness matrices) and in different languages. We also provide an extension to the vector case in arbitrary dimension, where the algorithm is applied to the elastic stiffness matrix with variable coefficients, in $2 \mathrm{D}$ and $3 \mathrm{D}$.

For space considerations, we restrict ourselves in this paper to $\mathbb{P}_{1}$ Lagrange finite elements. However, in the appendix we show that with slight modification, the algorithm is valid for piecewise polynomials of higher order.

These algorithms can be efficiently implemented in many languages if the language has a sparse matrix implementation. For the OptV1, OptV2, OptV and OptVS versions, a particular sparse matrix constructor is also needed (see Section 3) and these versions require that the language supports element-wise array operations. Examples of languages for which we obtained an efficient implementation of these algorithms are

- Matlab,

- Octave,

- Python with NumPy and SciPy modules,

- Scilab,

- Thrust and Cusp, C++ libraries for CUDA

This paper is organized as follows: in Section 2 we define two examples of finite element matrices. Then we introduce the notation associated to the mesh and to the algorithmic language used in this article. In Section 3 we give the classical and OptV1 algorithms. In Section 4 we present the vectorized OptV2 and OptV algorithms for a generic sparse matrix and $\mathbb{P}_{1}$ finite elements, with the application to the assemblies of the matrices of Section 2 A similar version called OptVS for symmetric matrices is also given. A first step towards finite elements of higher order is deferred to Appendix B. In Section 5 we consider the extension to the vector case with an application to linear elasticity. In Section 6. benchmark results illustrate the performance of the algorithms in the Matlab, Octave and Python languages. First, we show a comparison between the classical, OptV1, OptV2, OptV and OptVS versions. Then we compare the performances of the OptVS version to those obtained with a compiled language (using SuiteSparse [10] in C language), the latter being well-known to run at high speed and serving as a reference. A comparison is also given with FreeFEM ++18 as a simple and reliable finite element software. We also show in Matlab and Octave a comparison of the OptVS algorithm and the codes given in $[2,3,17,34$.

All the computations are done on our reference computen 2 with the releases R2014b for Matlab, 3.8.1 for Octave, 3.4.0 for Python and 3.31 for FreeFEM++. The Matlab/Octave and Python codes may be found in [7].

\footnotetext{
22 x Intel Xeon E5-2630v2 (6 cores) at 2.60Ghz, 64Go RAM
} 


\section{Statement of the problem and notation}

In this article we consider the assembly of the standard sparse matrices (e.g. weighted mass, stiffness and elastic stiffness matrices) arising from the $\mathbb{P}_{1}$ finite element discretization of partial differential equations (see e.g. [5, 33) in a bounded domain $\Omega$ of $\mathbb{R}^{d}(d \geq 1)$.

We suppose that $\Omega$ is equipped with a mesh $\mathcal{T}_{h}$ (locally conforming) as described in Table 2.1. We suppose that the elements belonging to the mesh are $d$ simplices . We introduce the finite dimensional space $X_{h}^{1}=\left\{v \in \mathcal{C}^{0}\left(\overline{\Omega_{h}}\right), v_{\mid K} \in\right.$ $\left.\mathbb{P}_{1}(K), \forall K \in \mathcal{T}_{h}\right\}$ where $\Omega_{h}=\bigcup_{K \in \mathcal{T}_{h}} K$ and $\mathbb{P}_{1}(K)$ denotes the space of all polynomials over $K$ and of total degree less than or equal to 1 . Let $\mathrm{q}^{j}$, $j=1, \ldots, \mathrm{n}_{\mathrm{q}}$ be a vertex of $\Omega_{h}$, with $\mathrm{n}_{\mathrm{q}}=\operatorname{dim}\left(X_{h}^{1}\right)$. The space $X_{h}^{1}$ is spanned by the $\mathbb{P}_{1}$ Lagrange basis functions $\left\{\varphi_{i}\right\}_{i \in\left\{1, \ldots, \mathrm{n}_{\mathrm{q}}\right\}}$ in $\mathbb{R}^{d}$, where $\varphi_{i}\left(\mathrm{q}^{j}\right)=\delta_{i j}$, with $\delta_{i j}$ the Kronecker delta.

We consider two examples of finite element matrices: the weighted mass matrix $\mathbb{M}^{[w]}$, with $w \in L^{\infty}(\Omega)$, defined by

$$
\mathbb{M}_{i, j}^{[w]}=\int_{\Omega_{h}} w \varphi_{j} \varphi_{i} d \mathrm{q}, \quad \forall(i, j) \in\left\{1, \ldots, \mathrm{n}_{\mathrm{q}}\right\}^{2},
$$

and the stiffness matrix $\mathbb{S}$ given by

$$
\mathbb{S}_{i, j}=\int_{\Omega_{h}}\left\langle\nabla \varphi_{j}, \nabla \varphi_{i}\right\rangle d \mathrm{q}, \quad \forall(i, j) \in\left\{1, \ldots, \mathrm{n}_{\mathrm{q}}\right\}^{2} .
$$

Note that on the $k$-th element $K=T_{k}$ of $\mathcal{T}_{h}$ we have

$$
\forall \alpha \in\{1, \ldots, d+1\}, \quad \varphi_{i \mid T_{k}}=\lambda_{\alpha}, \text { with } i=\operatorname{me}(\alpha, k),
$$

where $\left(\lambda_{\alpha}\right)_{\alpha \in\{1, \ldots, d+1\}}$ are the barycentric coordinates (i.e the local $\mathbb{P}_{1}$ Lagrange basis functions) of $K$, and me is the connectivity array (see Table 2.1). The matrices $\mathbb{M}^{[w]}$ and $\mathbb{S}$ can be assembled efficiently with a vectorized algorithm proposed in Section 4, which uses the following formula (see e.g. [31])

$$
\int_{K} \prod_{i=1}^{d+1} \lambda_{i}^{n_{i}} d \mathrm{q}=d !|K| \frac{\prod_{i=1}^{d+1} n_{i} !}{\left(d+\sum_{i=1}^{d+1} n_{i}\right) !}
$$

where $|K|$ is the volume of $K$ and $n_{i} \in \mathbb{N}$.

Remark 2.1 The (non-vectorized or vectorized) finite element assembly algorithms presented in this article may be adapted to compute matrices associated to the bilinear form (1.1).

Remark 2.2 These algorithms apply to finite element methods of higher order. Indeed, one can express the $\mathbb{P}_{k}$-Lagrange basis functions $(k \geq 2)$ as polynomials in $\lambda_{i}$ variable and then use formula (2.4). In Appendix D we give a first step to obtain a vectorized algorithm for $\mathbb{P}_{k}$ finite elements. 
In the remainder of this article, we will use the following notations to describe the triangulation $\mathcal{T}_{h}$ of $\Omega$ :

\begin{tabular}{lccl}
\hline name & type & dimension & description \\
\hline$d$ & integer & 1 & dimension of simplices of $\mathcal{T}_{h}$ \\
$\mathrm{n}_{\mathrm{q}}$ & integer & 1 & number of vertices of $\mathcal{T}_{h}$ \\
$\mathrm{n}_{\text {me }}$ & integer & 1 & number of mesh elements in $\mathcal{T}_{h}$ \\
$\mathrm{q}$ & double & $d \times \mathrm{n}_{\mathrm{q}}$ & array of vertex coordinates \\
me & integer & $(d+1) \times \mathrm{n}_{\text {me }}$ & connectivity array \\
vols & double & $1 \times \mathrm{n}_{\mathrm{me}}$ & array of simplex volumes \\
\hline
\end{tabular}

Table 2.1: Data structure associated to the mesh $\mathcal{T}_{h}$

In Table 2.1, for $\nu \in\{1, \ldots, d\}, \mathrm{q}(\nu, j)$ represents the $\nu$-th coordinate of the $j$-th vertex, $j \in\left\{1, \ldots, \mathrm{n}_{\mathrm{q}}\right\}$. The $j$-th vertex will be also denoted by $\mathrm{q}^{j}$. The term $\operatorname{me}(\beta, k)$ is the storage index of the $\beta$-th vertex of the $k$-th element, in the array $q$, for $\beta \in\{1, \ldots, d+1\}$ and $k \in\left\{1, \ldots, \mathrm{n}_{\text {me }}\right\}$.

We also provide below some common functions and operators of the vectorized algorithmic language used in this article which generalize the operations on scalars to higher dimensional arrays, matrices and vectors:

$\begin{array}{ll}\mathbb{A} \leftarrow \mathbb{B} & \begin{array}{l}\text { Assignment } \\ \text { matrix multiplication, }\end{array} \\ \mathbb{A} * \mathbb{B} & \text { element-wise multiplication, } \\ \mathbb{A} \cdot / \mathbb{B} & \text { element-wise division, } \\ \mathbb{A}(:) & \text { all the elements of } \mathbb{A}, \text { regarded as a single column. } \\ {[,]} & \text { Horizontal concatenation, } \\ {[;]} & \text { Vertical concatenation, } \\ \mathbb{A}(:, J) & J \text {-th column of } \mathbb{A}, \\ \mathbb{A}(I,:) & I \text {-th row of } \mathbb{A}, \\ \operatorname{SUM}(\mathbb{A}, \operatorname{dim}) & \text { sums along the dimension dim, } \\ \mathbb{I}_{n} & n \text {-by- } n \text { identity matrix, } \\ \mathbb{1}_{m \times n}\left(\text { or } \mathbb{1}_{n}\right) & m \text {-by- } n \text { (or } n \text {-by- } n \text { ) matrix or sparse matrix of ones, } \\ \mathbb{O}_{m \times n}\left(\text { or } \mathbb{O}_{n}\right) & m \text {-by- } n \text { (or } n \text {-by- } n \text { ) matrix or sparse matrix of zeros, } \\ \text { ONES }\left(n_{1}, n_{2}, \ldots, n_{\ell}\right) & \ell \text { dimensional array of ones, } \\ \text { ZEROS }\left(n_{1}, n_{2}, \ldots, n_{\ell}\right) & \ell \text { dimensional array of zeros. }\end{array}$

\section{Standard finite element assemblies}

In this section we consider the $\mathbb{P}_{1}$ finite element assembly of a generic $n_{\mathrm{q}}$-by- $\mathrm{n}_{\mathrm{q}}$ sparse matrix $\mathbb{M}$ with its corresponding $(d+1)$-by- $(d+1)$ local matrix $\mathbb{E}$ (also denoted by $\mathbb{E}(K)$ when referring to an element $\left.K \in \mathcal{T}_{h}\right)$. For $K=T_{k}$, the $(\alpha, \beta)$-th entry of $\mathbb{E}\left(T_{k}\right)$ is denoted by $e_{\alpha, \beta}^{k}$.

In Algorithm 3.1, we recall the classical finite element assembly method for calculating $\mathbb{M}$. In this algorithm, an $\mathrm{n}_{\mathrm{q}}-\mathrm{by}-\mathrm{n}_{\mathrm{q}}$ sparse matrix $\mathbb{M}$ is first declared, then the contribution of each element $T_{k} \in \mathcal{T}_{h}$, given by a function ElemMat, is added to the matrix $\mathbb{M}$. These successive operations are very expensive due to a suboptimal use of the sparse function. 
A first optimized, non-vectorized, version (called OptV1), suggested in [9], is based on the use of the sparse function:

$$
\mathrm{M} \leftarrow \operatorname{sparse}(\mathrm{Ig}, \mathrm{Jg}, \mathrm{Kg}, \mathrm{m}, \mathrm{n}) ;
$$

This command returns an $\mathrm{m} \times \mathrm{n}$ sparse matrix $M$ such that

$$
\mathrm{M}(\operatorname{Ig}(\mathrm{k}), \mathrm{Jg}(\mathrm{k})) \leftarrow \mathrm{M}(\operatorname{Ig}(\mathrm{k}), \mathrm{Jg}(\mathrm{k}))+\mathrm{Kg}(\mathrm{k}) .
$$

The vectors $\mathrm{Ig}$, Jg and $\mathrm{Kg}$ have the same length. The zero elements of $\mathrm{K}$ are not taken into account and the elements of $\mathrm{Kg}$ having the same indices in $\mathrm{Ig}$ and $\mathrm{Jg}$ are summed.

Examples of languages containing a sparse function are given below

- Python (scipy.sparse module) :

$$
\mathrm{M}=\text { sparse. }<\text { format }>\operatorname{matrix}((\mathrm{Kg},(\mathrm{Ig}, \mathrm{Jg})) \text {, shape }=(\mathrm{m}, \mathrm{n}))
$$

where <format> is the sparse matrix format (e.g. csc, csr, lil, ...),

- Matlab : M=sparse ( $\mathrm{Ig}, \mathrm{Jg}, \mathrm{Kg}, \mathrm{m}, \mathrm{n})$, only csc format,

- Octave : $M=\operatorname{sparse}(\mathrm{Ig}, \mathrm{Jg}, \mathrm{Kg}, \mathrm{m}, \mathrm{n})$, only csc format,

- Scilab : M=sparse ( $[\mathrm{Ig}, \mathrm{Jg}], \mathrm{Kg},[\mathrm{m}, \mathrm{n}]$ ), only row-by-row format.

- C with SuiteSparse 10 ]

- CUDA with Thrust [29] and Cusp [28] libraries

The OptV1 version consists in computing and storing all elementary contributions first and then using them to generate the sparse matrix $\mathbb{M}$. The main idea is to create three global 1d-arrays $\boldsymbol{K}_{g}, \boldsymbol{I}_{g}$ and $\boldsymbol{J}_{g}$ of length $(d+1)^{2} \mathrm{n}_{\text {me }}$, which store the local matrices as well as the position of their elements in the global matrix as shown on Figure 3.1. To create the arrays $\boldsymbol{K}_{g}, \boldsymbol{I}_{g}$ and $\boldsymbol{J}_{g}$, we define three local arrays $\boldsymbol{K}_{k}^{e}, \boldsymbol{I}_{k}^{e}$ and $\boldsymbol{J}_{k}^{e}$ of length $(d+1)^{2}$ obtained from the $(d+1)$-by- $(d+1)$ local matrix $\mathbb{E}\left(T_{k}\right)$ as follows:

$K_{k}^{e} \quad$ : elements of the matrix $\mathbb{E}\left(T_{k}\right)$ stored column-wise,

$\boldsymbol{I}_{k}^{e} \quad$ : global row indices associated to the elements stored in $\boldsymbol{K}_{k}^{e}$,

$\boldsymbol{J}_{k}^{e} \quad$ : global column indices associated to the elements stored in $\boldsymbol{K}_{k}^{e}$.

Using $\boldsymbol{K}_{k}^{e}, \boldsymbol{I}_{k}^{e}, \boldsymbol{J}_{k}^{e}$ and a loop over the mesh elements $T_{k}$, one may calculate the global arrays $\boldsymbol{I}_{g}, \boldsymbol{J}_{g}$ and $\boldsymbol{K}_{g}$. The corresponding OptV1 algorithm is given in Algorithm 3.2 .

Numerical experiments in Section 6.1 and in Tables A.3 and A.4 show that the OptV1 algorithm is more efficient than the classical one. The inefficiency of the classical (base) version compared to the OptV1 version is mainly due to the repetition of element insertions into the sparse structure and to some dynamic reallocation troubles that may also occur.

However, the OptV1 algorithm still uses a loop over the elements. To improve the efficiency of this algorithm, we propose in the next section other optimized versions, in a vectorized form: the main loop over the elements, which increases with the size of the mesh, is vectorized. The other loops (which are independent of the mesh size and with few iterations) will not necessarily be vectorized. 


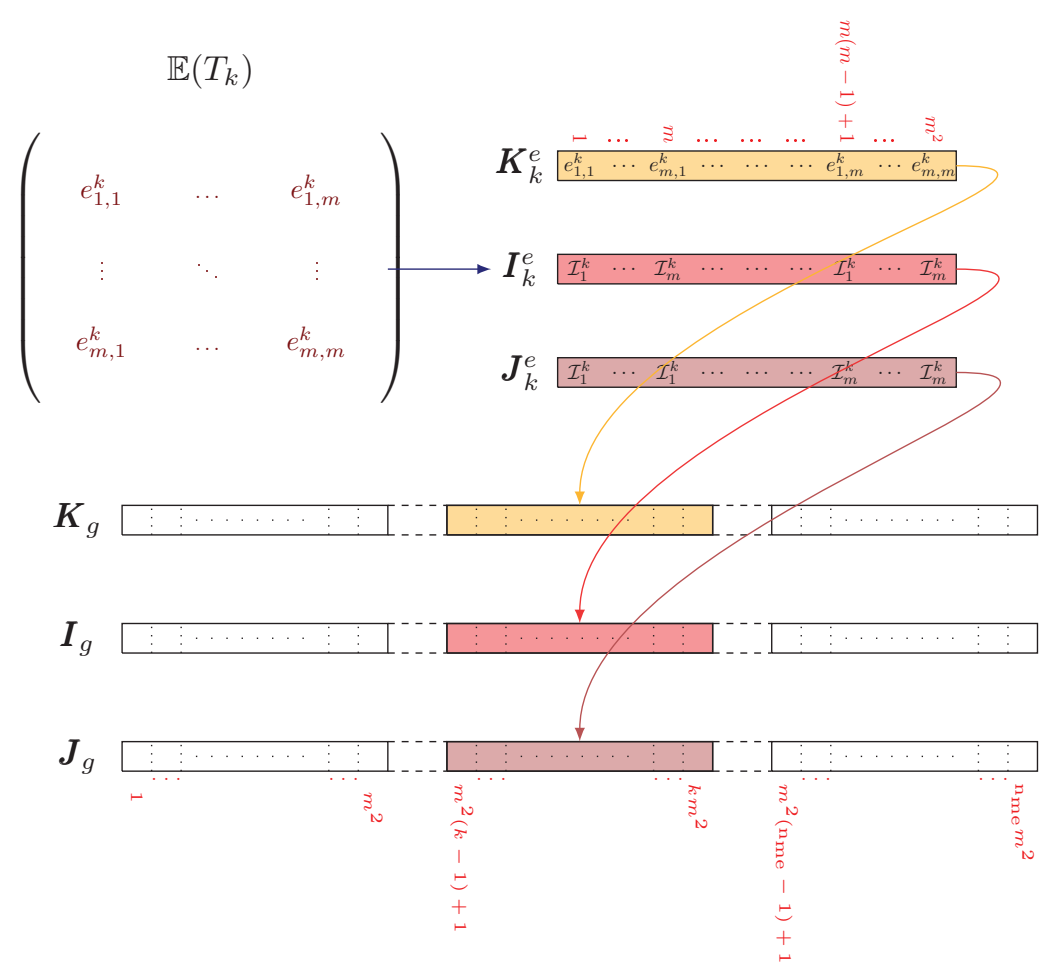

Fig. 3.1: Insertion of a local matrix into global 1d-arrays - OptV1 version, where $m=d+1, \mathcal{I}_{l}^{k}=\operatorname{me}(l, k)$.

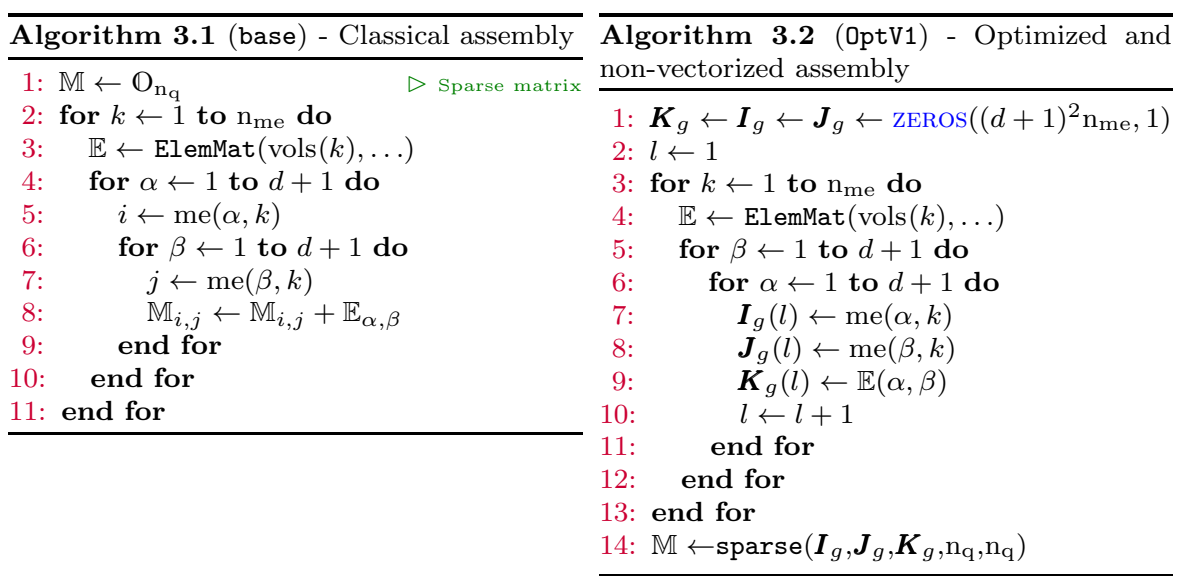




\section{Optimized finite element assembly}

In this section we present optimized algorithms, only available in vector languages. In the first algorithm, OptV2, the idea is to vectorize the main loop over the elements by defining the two-dimensional arrays $\mathbb{K}_{g}, \mathbb{I}_{g}$ and $\mathbb{J}_{g}$ of size $(d+1)^{2}$-by- $\mathrm{n}_{\text {me }}$ which store all the local matrices as well as their positions in the global matrix. Then, as for the OptV1 version, the matrix assembly is obtained with the sparse function:

$$
\mathrm{M} \leftarrow \operatorname{sparse}\left(\mathbb{I}_{g}(:), \mathbb{J}_{g}(:), \mathbb{K}_{g}(:), \mathrm{n}_{\mathrm{q}}, \mathrm{n}_{\mathrm{q}}\right) ;
$$

A non-vectorized approach inspired by OptV1 is as follows: for each mesh element $T_{k}$, the $k$-th column of the global arrays $\mathbb{K}_{g}, \mathbb{I}_{g}$ and $\mathbb{J}_{g}$ is filled with the local arrays $\boldsymbol{K}_{k}^{e}, \boldsymbol{I}_{k}^{e}, \boldsymbol{J}_{k}^{e}$ respectively, as shown in Figure 4.1.

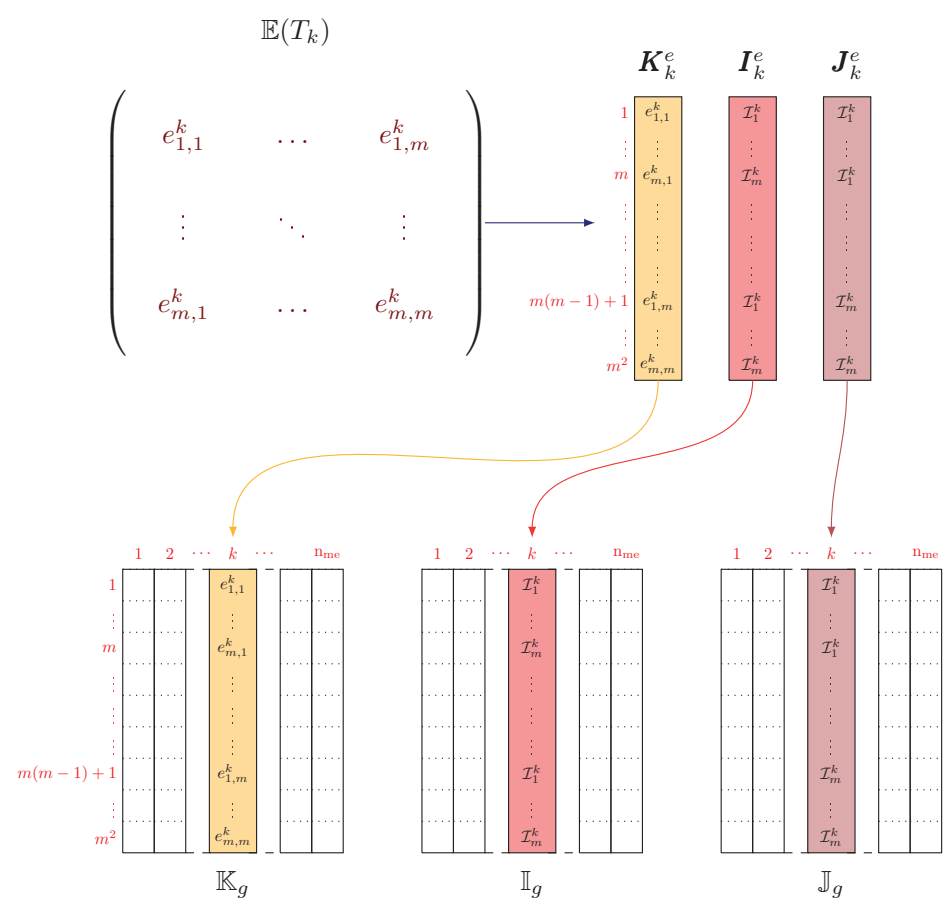

Fig. 4.1: Insertion of a local matrix into global 2D-arrays

Thus, $\mathbb{K}_{g}, \mathbb{I}_{g}$ and $\mathbb{J}_{g}$ are defined by: $\forall k \in\left\{1, \ldots, \mathrm{n}_{\text {me }}\right\}, \forall l \in\left\{1, \ldots,(d+1)^{2}\right\}$,

$$
\mathbb{K}_{g}(l, k)=\boldsymbol{K}_{k}^{e}(l), \quad \mathbb{I}_{g}(l, k)=\boldsymbol{I}_{k}^{e}(l), \quad \mathbb{J}_{g}(l, k)=\boldsymbol{J}_{k}^{e}(l) .
$$

A natural way to calculate these three arrays is column-wise. In that case, for each array one needs to compute $\mathrm{n}_{\text {me }}$ columns. 
The OptV2 method consists in calculating these arrays row-wise. In that case, for each array one needs to calculate $(d+1)^{2}$ rows (where $d$ is independent of the number of mesh elements). This vectorization method is represented in Figure 4.2
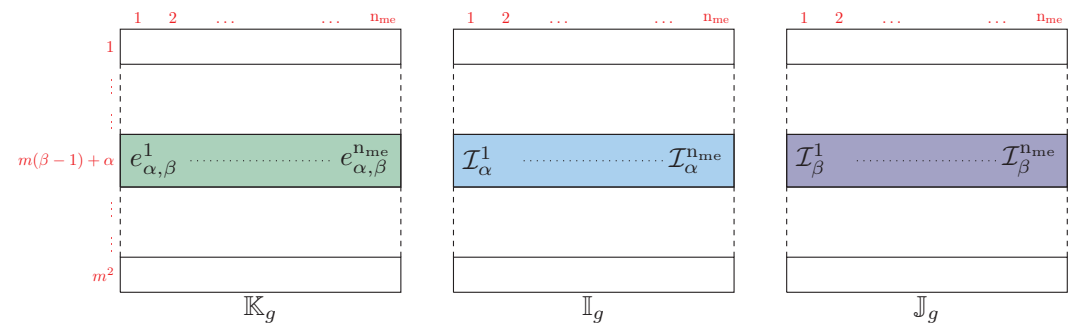

Fig. 4.2: Row-wise operations on global 2D-arrays

We first suppose that for $\alpha$ and $\beta$ fixed, we can vectorize the computation of $e_{\alpha, \beta}^{k}$, for all $k \in\left\{1, \ldots, \mathrm{n}_{\mathrm{me}}\right\}$. This vectorization procedure, denoted by $\operatorname{vecELem}(\alpha, \beta, \ldots)$, returns a $1 \mathrm{~d}$-array containing these $\mathrm{n}_{\text {me }}$ values. We will describe it in detail for some examples in Sections 4.1 and 4.2. Then we obtain the following algorithm

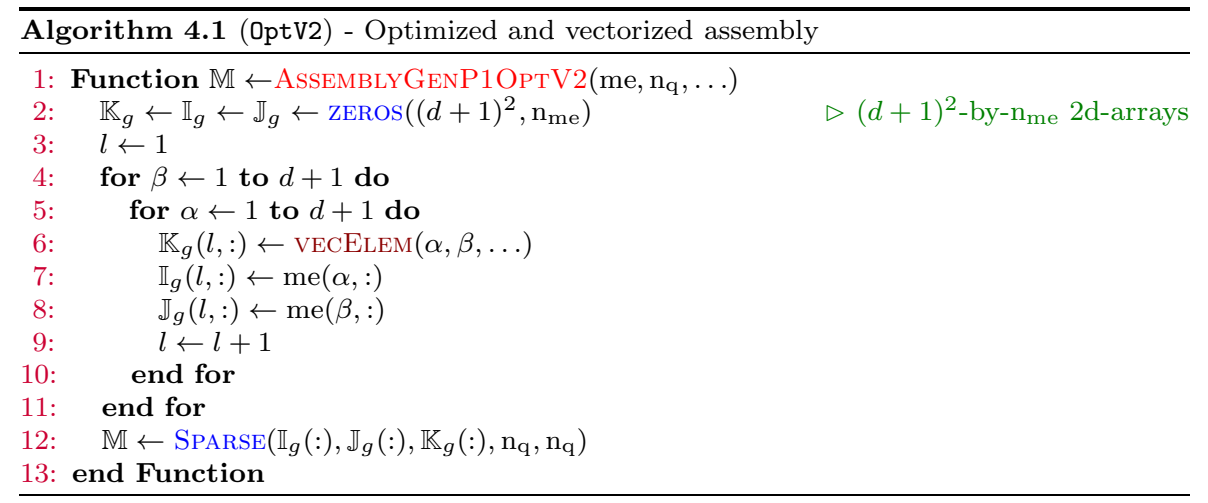

Algorithm 4.1 is efficient in terms of computation time (see Section 6.1). However it is memory consuming due to the size of the arrays $\mathbb{I}_{g}, \mathbb{J}_{g}$ and $\mathbb{K}_{g}$. Thus a variant (see [3,23] for dimension 2 or 3 in Matlab) consists in using the sparse command inside the loops (i.e. for each component of all element matrices). This method, called OptV, is given in Algorithm 4.2. 


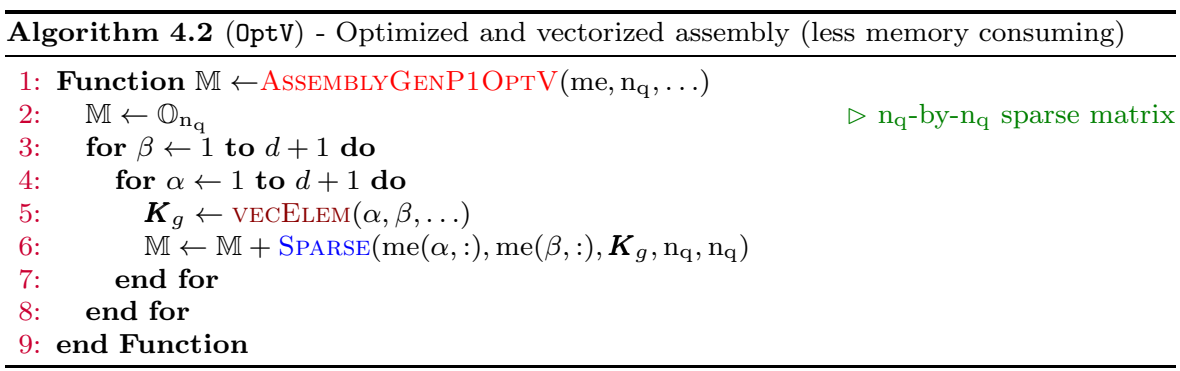

For a symmetric matrix, the performance can be improved by using a symmetrized version of OptV (called OptVS), given in Algorithm 4.3. More precisely, in the lines 3.8 of this algorithm, we build a non-triangular sparse matrix which contains the contributions of the strictly upper parts of all the element matrices. In line 9 the strictly lower part contributions are added using the symmetry of the element matrices. Then in lines 10,13 the contributions of the diagonal parts of the element matrices are added.

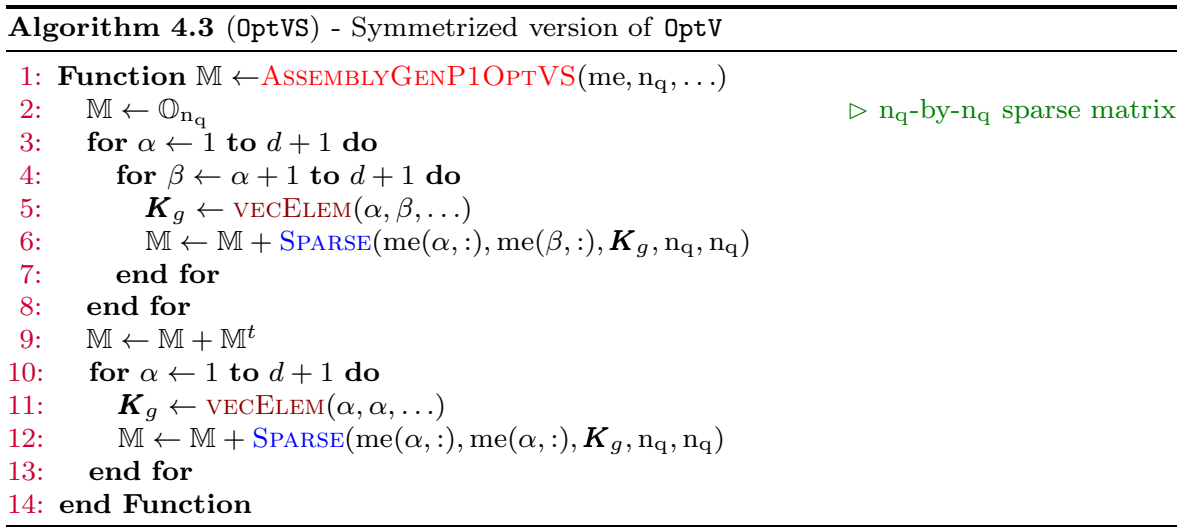

In the following, our objective is to show using examples how to vectorize the computation of $\boldsymbol{K}_{g}$ (i.e. how to obtain the vECELEM function in algorithms OptV2, OptV and OptVS). More precisely for the examples derived from (1.1), the calculation of $\boldsymbol{K}_{g}$ only depends on the local basis functions and/or their gradients and one may need to calculate them on all mesh elements. For $\mathbb{P}_{1}$ finite elements, these gradients are constant on each $d$-simplex $K=T_{k}$. Let $\mathbb{G}$ be the $3 \mathrm{D}$ array of size $\mathrm{n}_{\text {me-by- }}(d+1)$-by- $d$ defined by

$$
\mathbb{G}(k, \alpha,:)=\nabla \varphi_{\alpha}^{k}(\mathrm{q}), \quad \forall \alpha \in\{1, \ldots, d+1\}, \forall k \in\left\{1, \ldots, \mathrm{n}_{\text {me }}\right\} .
$$

In Appendix C, we give a vectorized function called GradientVec (see Algorithm C.1] which computes $\mathbb{G}$ in arbitrary dimension. Once the gradients are computed, the local matrices are calculated using the formula (2.4). For simplicity, in the following we consider the OptV version. The vectorization of the computation of $\boldsymbol{K}_{g}$ is shown using the two examples introduced in Section 2. 
4.1 Weighted mass matrix assembly

The local weighted mass matrix $\mathbb{M}^{[w], e}(K)$ is given by

$$
\mathbb{M}_{\alpha, \beta}^{[w], e}(K)=\int_{K} w \lambda_{\beta} \lambda_{\alpha} d \mathrm{q}, \quad \forall(\alpha, \beta) \in\{1, \ldots, d+1\}^{2},
$$

with $w \in L^{\infty}(\Omega)$. Generally, this matrix cannot be computed exactly and one has to use a quadrature formula. In the following, we choose to approximate $w$ by $w_{h}=\pi_{K}^{1}(w)$ where $\pi_{K}^{1}(w)=\sum_{\gamma=1}^{d+1} w\left(\mathrm{q}^{\gamma}\right) \lambda_{\gamma}$ is the $\mathbb{P}_{1}$ Lagrange interpolation of $w$. Then using (2.4), we have the quadrature formula for (4.2)

$$
\int_{K} \pi_{K}^{1}(w) \lambda_{\alpha} \lambda_{\beta} d \mathrm{q}=\frac{d !}{(d+3) !}|K|\left(1+\delta_{\alpha, \beta}\right)\left(w^{s}+w\left(\mathrm{q}^{\alpha}\right)+w\left(\mathrm{q}^{\beta}\right)\right),
$$

where $w^{s}=\sum_{\gamma=1}^{d+1} w\left(\mathrm{q}^{\gamma}\right)$. Using (4.3) we vectorize the assembly of the approximate weighted mass matrix (2.1) as shown in Algorithm 4.4

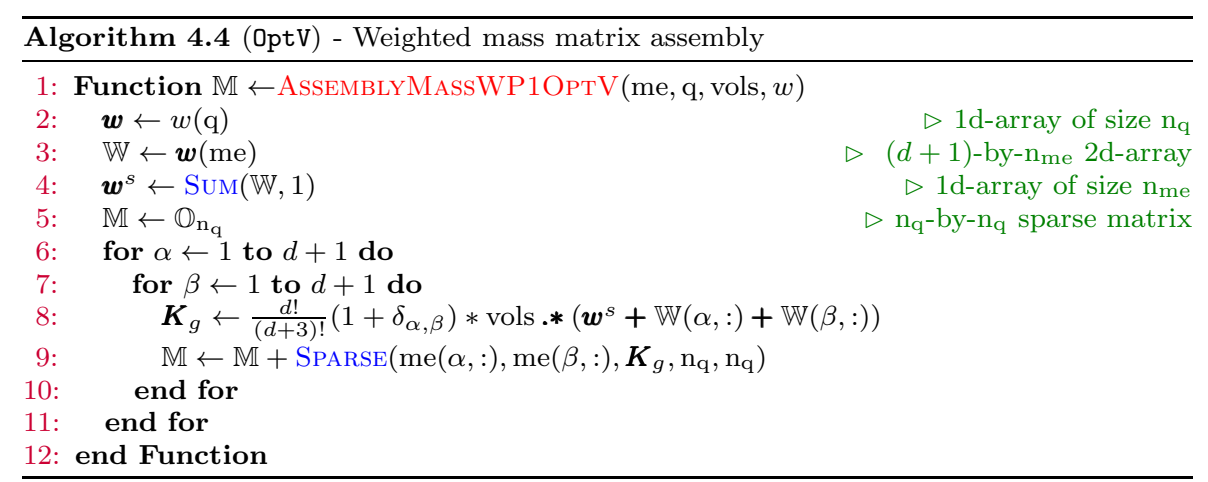

Line 8 of Algorithm 4.4 corresponds to the vectorization of formula (4.3) and is carried out as follows: first we set $\boldsymbol{w} \in \mathbb{R}^{\mathrm{n}_{\mathrm{q}}}$ such that $\boldsymbol{w}(i)=w\left(\mathrm{q}^{i}\right), 1 \leq i \leq \mathrm{n}_{\mathrm{q}}$, or in a vectorized form $\boldsymbol{w} \leftarrow w(\mathrm{q})$. Then we compute the array $\mathbb{W}$ of size $(d+1)$-by- $\mathrm{n}_{\text {me }}$ containing, for each $d$-simplex, the values of $w$ at its vertices: $\mathbb{W}(\alpha, k)=w\left(\mathrm{q}^{\mathrm{me}(\alpha, k)}\right)$ or in vectorized form $\mathbb{W} \leftarrow \boldsymbol{w}(\mathrm{me})$. We now calculate $\boldsymbol{w}^{s} \in \mathbb{R}^{n_{\text {me }}}$ which contains, for each $d$-simplex, the sum of the values of $w$ at its vertices, i.e. we sum $\mathbb{W}$ over the rows and obtain line 4 of Algorithm 4.4 Then, formula (4.3) may be vectorized to obtain line 8 in Algorithm 4.4.

Remark 4.1 Note that formula (4.3) is exact if $w$ is a polynomial of degree 1 on $K$. Moreover, if $w$ is constant, we get the mass matrix (up to the constant $w$ ). Other quadrature rules could be used to approximate the integral in (4.2) without changing the principle of Algorithm 4.4.

Remark 4.2 Algorithm 4.4 can be applied to meshes composed of $n$-simplices (for $n \leq d$ ) and may be used to compute Neumann or Robin boundary terms. 
4.2 Stiffness matrix assembly

The local stiffness matrix $\mathbb{S}^{e}(K)$ is given, for all $(\alpha, \beta) \in\{1, \ldots, d+1\}^{2}$, by

$$
\mathbb{S}_{\alpha, \beta}^{e}(K)=\int_{K}\left\langle\nabla \lambda_{\beta}, \nabla \lambda_{\alpha}\right\rangle d \mathrm{q}=|K|\left\langle\nabla \lambda_{\beta}, \nabla \lambda_{\alpha}\right\rangle
$$

To obtain the right-hand side of (4.4) we use the fact that the gradients of the local basis functions are constant on each $d$-simplex. The gradients are computed with the vectorized function GradientVec of Algorithm C.1 Then the vectorized assembly Algorithm 4.5 easily follows.

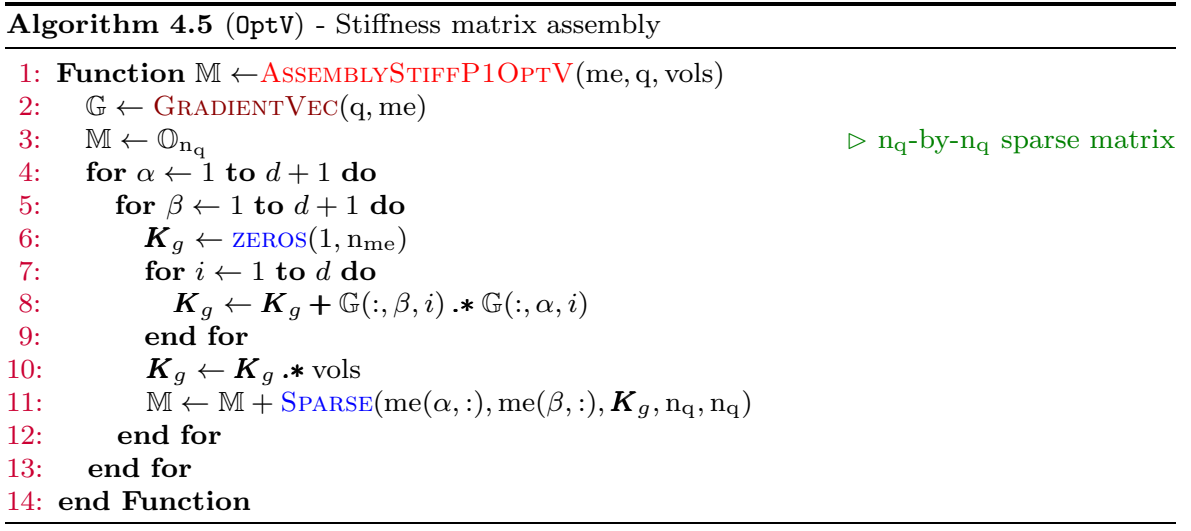

We will now adapt these methods to the vector case with an application to the assembly of the elastic stiffness matrix in two and three dimensions.

\section{Extension to the vector case}

In this section we present an extension of Algorithms 4.1 and 4.2 to the vector case, i.e for a system of $m(m>1)$ partial differential equations such as in elasticity. First, we need to introduce some notation: the space $\left(X_{h}^{1}\right)^{m}$ (where $X_{h}^{1}$ is defined in Section 2), is of dimension $\mathrm{n}_{\mathrm{dof}}=m \mathrm{n}_{\mathrm{q}}$ and spanned by the vector basis functions $\left\{\boldsymbol{\psi}_{l, i}\right\}_{1 \leq i \leq \mathrm{n}_{\mathrm{q}}}$, given by

$$
\begin{array}{r}
\boldsymbol{\psi}_{l, i}=\varphi_{i} \boldsymbol{e}_{l}, \\
\text {, }
\end{array}
$$

where $\left\{\boldsymbol{e}_{1}, \cdots, \boldsymbol{e}_{m}\right\}$ is the standard basis of $\mathbb{R}^{m}$. The alternate numbering is chosen for the basis functions. We use either $\boldsymbol{\psi}_{l, i}$ or $\boldsymbol{\psi}_{s}$ with $s=(i-1) m+l$ to denote them. We will consider the assembly of a generic sparse matrix of dimension $\mathrm{n}_{\text {dof }}-$ by- $\mathrm{n}_{\text {dof }}$ defined by

$$
\mathbb{H}_{r, s}=\int_{\Omega_{h}} \mathcal{H}\left(\boldsymbol{\psi}_{s}, \boldsymbol{\psi}_{r}\right) d \mathrm{q}
$$


where $\mathcal{H}$ is a bilinear differential operator of order one.

As in the scalar case, in order to vectorize the assembly of the matrix, one has to vectorize the computation of the local matrices. To define the local matrix, we introduce the following notation: on the $k$-th element $K=T_{k}$ of $\mathcal{T}_{h}$ we denote by $\left\{\boldsymbol{\lambda}_{l, \alpha}\right\}_{1 \leq l \leq m}$ the $\mathrm{n}_{\mathrm{dfe}}=m(d+1)$ local basis functions defined by

$$
\lambda_{l, \alpha}=\lambda_{\alpha} \boldsymbol{e}_{l}
$$

We also use notation $\boldsymbol{\lambda}_{i}$ with $i=(\alpha-1) m+l$ to denote $\boldsymbol{\lambda}_{l, \alpha}$. By construction, we have $\forall l \in\{1, \ldots, m\}, \forall \alpha \in\{1, \ldots, d+1\}$

$$
\psi_{l, \operatorname{me}(\alpha, k)}=\lambda_{l, \alpha} \text { on } K=T_{k}
$$

Thus, the local matrix $\mathbb{H}^{e}$ on the $d$-simplex $K$ is of size $\mathrm{n}_{\mathrm{dfe}}-\mathrm{by}-\mathrm{n}_{\mathrm{dfe}}$, and is given by

$$
\mathbb{H}_{i, j}^{e}=\int_{K} \mathcal{H}\left(\boldsymbol{\lambda}_{j}, \boldsymbol{\lambda}_{i}\right) d \mathrm{q}
$$

Then, a classical non-vectorized algorithm is given in Algorithm5.1, The function ElemH is used to calculate the matrix $\mathbb{H}^{e}$ for a given $d$-simplex $K$. As in the scalar case, the vectorized assembly algorithm is based on the use of a function called $\mathrm{VECHE}$ which returns the values corresponding to the $(i, j)$-th entry $($ with $(i, j)=(m(\alpha-1)+l, m(\beta-1)+n))$ of the local matrices $\mathbb{H}^{e}(K)$, for all $K \in \mathcal{T}_{h}$ and for all $l, \alpha, n, \beta$. We suppose that this function can be vectorized. Then we obtain the OptV2 vectorized assembly of the matrix $\mathbb{H}$ given in Algorithm 5.2

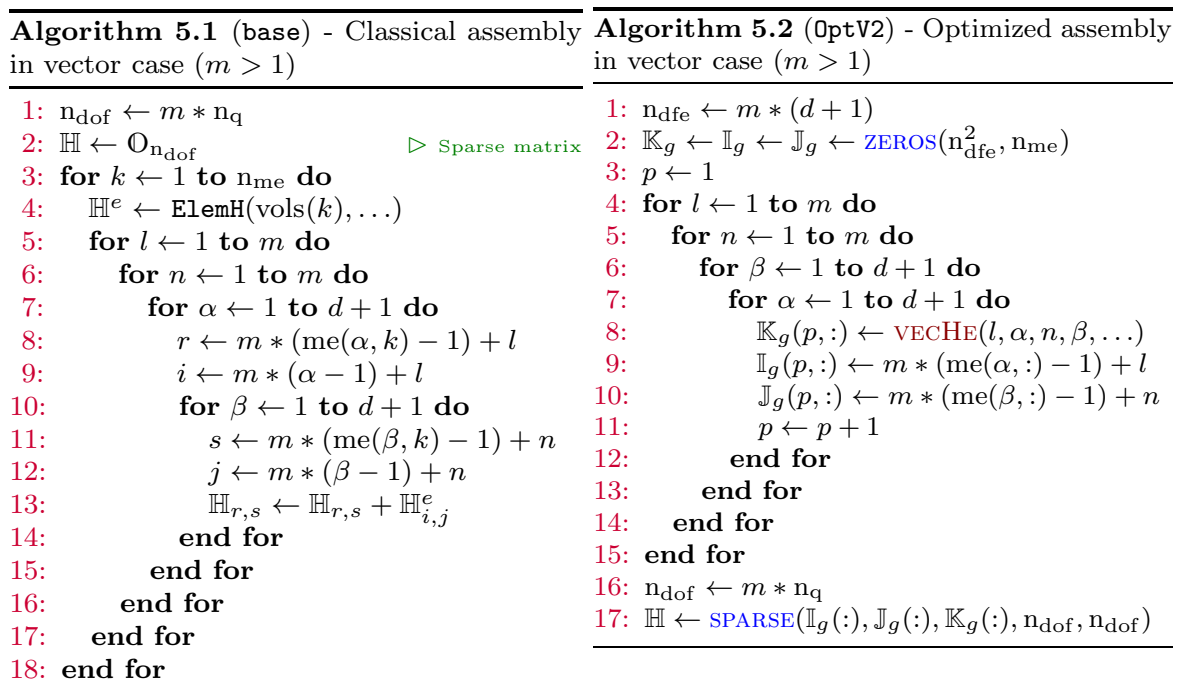


As in Section 4, although Algorithm5.2 is efficient in terms of computation time, it is memory consuming due to the size of the arrays $\mathbb{I}_{g}, \mathbb{J}_{g}$ and $\mathbb{K}_{g}$. Thus a variant consists in using the sparse command inside the loops, which leads to the extension of the $0 \mathrm{ptV}$ algorithm to the vector case, given in Algorithm 5.3

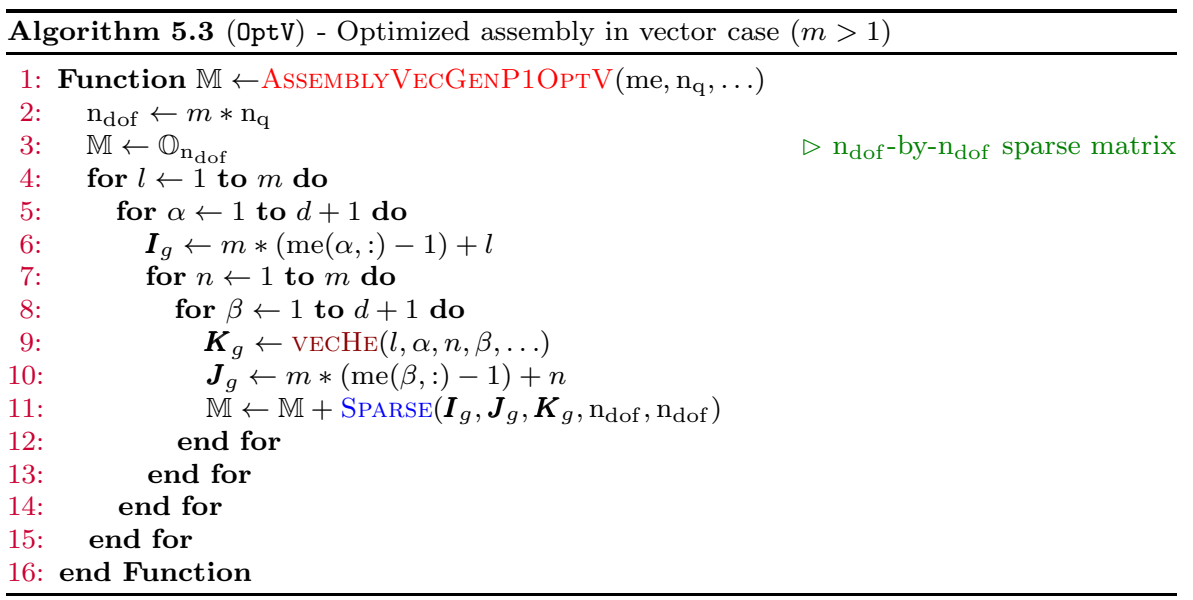

For a symmetric matrix, the performance can be improved by using a symmetrized version of Algorithm 5.3 (as in Section 4), given in Algorithm C.2

In the following the vectorized function $\mathrm{VECHE}$ is detailed for the elastic stiffness matrix in $2 \mathrm{D}$ and $3 \mathrm{D}$.

\subsection{Elastic stiffness matrix assembly}

Here we consider sufficiently regular vector fields $\boldsymbol{u}=\left(u_{1}, \ldots, u_{d}\right): \Omega \rightarrow \mathbb{R}^{d}$, with the associated discrete space $\left(X_{h}^{1}\right)^{d}, d=2$ or 3 (i.e. $m=d$ in that case).

We consider the elastic stiffness matrix arising in linear elasticity when Hooke's law is used and the material is isotropic, under small strain hypothesis (see for example [11). This sparse matrix $\mathbb{K}$ is defined by

$$
\mathbb{K}_{l, n}=\int_{\Omega_{h}} \underline{\boldsymbol{\epsilon}}^{t}\left(\boldsymbol{\psi}_{n}\right) \mathbb{C} \underline{\boldsymbol{\epsilon}}\left(\boldsymbol{\psi}_{l}\right) d \mathrm{q}, \quad \forall(l, n) \in\left\{1, \ldots, \mathrm{n}_{\mathrm{dof}}\right\}^{2},
$$

where $\underline{\epsilon}$ is the linearized strain tensor given by

$$
\underline{\boldsymbol{\epsilon}}(\boldsymbol{u})=\frac{1}{2}\left(\boldsymbol{\nabla}(\boldsymbol{u})+\nabla^{t}(\boldsymbol{u})\right),
$$

with $\underline{\boldsymbol{\epsilon}}=\left(\epsilon_{11}, \epsilon_{22}, 2 \epsilon_{12}\right)^{t}$ in $2 \mathrm{D}$ and $\boldsymbol{\epsilon}=\left(\epsilon_{11}, \epsilon_{22}, \epsilon_{33}, 2 \epsilon_{12}, 2 \epsilon_{23}, 2 \epsilon_{13}\right)^{t}$ in $3 \mathrm{D}$, with $\epsilon_{i j}(\boldsymbol{u})=\frac{1}{2}\left(\frac{\partial u_{i}}{\partial x_{j}}+\frac{\partial u_{j}}{\partial x_{i}}\right)$. The elasticity tensor $\mathbb{C}$ depends on the Lamé 
parameters $\lambda$ and $\mu$ satisfying $\lambda+\mu>0$, and possibly variable in $\Omega$. For $d=2$ or $d=3$, the matrix $\mathbb{C}$ is given by

$$
\mathbb{C}=\left(\begin{array}{cc}
\lambda \mathbb{1}_{2}+2 \mu \mathbb{I}_{2} & \mathrm{O}_{2 \times 1} \\
\mathrm{O}_{1 \times 2} & \mu
\end{array}\right)_{3 \times 3}, \quad \mathbb{C}=\left(\begin{array}{cc}
\lambda \mathbb{1}_{3}+2 \mu \mathbb{I}_{3} & \mathrm{O}_{3 \times 3} \\
\mathrm{O}_{3 \times 3} & \mu \mathbb{I}_{3}
\end{array}\right)_{6 \times 6} .
$$

Formula (5.3) is related to the Hooke's law

$$
\underline{\boldsymbol{\sigma}}=\mathbb{C} \underline{\boldsymbol{\epsilon}},
$$

where $\underline{\sigma}$ is the elastic stress tensor.

The vectorization of the assembly of the elastic stiffness matrix (5.3) will be carried out as in Section 4, through the vectorization of the local elastic stiffness matrix $\mathbb{K}^{e}$ given for all $(i, j) \in\left\{1, \ldots, \mathrm{n}_{\mathrm{dfe}}\right\}^{2}$ by

$$
\mathbb{K}_{i, j}^{e}(K)=\int_{K} \underline{\boldsymbol{\epsilon}}^{t}\left(\boldsymbol{\lambda}_{j}\right) \mathbb{C} \underline{\boldsymbol{\epsilon}}\left(\boldsymbol{\lambda}_{i}\right) d \mathrm{q},
$$

or equivalently, using (5.2), we have for $1 \leq \alpha, \beta \leq d+1$ and $1 \leq l, n \leq m$

$$
\mathbb{K}_{i, j}^{e}(K)=\int_{K} \underline{\boldsymbol{\epsilon}}^{t}\left(\boldsymbol{\lambda}_{n, \beta}\right) \mathbb{C} \underline{\boldsymbol{\epsilon}}\left(\boldsymbol{\lambda}_{l, \alpha}\right) d \mathrm{q}
$$

with $i=(\alpha-1) d+l$ and $j=(\beta-1) d+n$. The vectorization of $\mathbb{K}^{e}$ is based on the following result:

Lemma 5.1 There exist two matrices $\mathbb{Q}^{n, l}$ and $\mathbb{S}^{n, l}$ of size $d$-by-d depending only on $n$ and $l$ such that

$$
\underline{\boldsymbol{\epsilon}}^{t}\left(\boldsymbol{\lambda}_{n, \beta}\right) \mathbb{C} \underline{\boldsymbol{\epsilon}}\left(\boldsymbol{\lambda}_{l, \alpha}\right)=\lambda\left\langle\nabla \lambda_{\beta}, \mathbb{Q}^{n, l} \nabla \lambda_{\alpha}\right\rangle+\mu\left\langle\nabla \lambda_{\beta}, \mathbb{S}^{n, l} \nabla \lambda_{\alpha}\right\rangle .
$$

The proof of Lemma 5.1 is given in Appendix B

Using (5.6) in (5.5), we have

$$
\mathbb{K}_{i, j}^{e}(K)=\left\langle\nabla \lambda_{\beta}, \mathbb{Q}^{n, l} \nabla \lambda_{\alpha}\right\rangle \int_{K} \lambda d \mathrm{q}+\left\langle\nabla \lambda_{\beta}, \mathbb{S}^{n, l} \nabla \lambda_{\alpha}\right\rangle \int_{K} \mu d \mathrm{q} .
$$

One possibility is to approximate the Lamé parameters $\lambda$ and $\mu$ by their $\mathbb{P}_{1}$ finite element interpolation $\pi_{K}^{1}(\lambda)$ and $\pi_{K}^{1}(\mu)$, respectively (we consider $\mathbb{P}_{1}$ instead of $\mathbb{P}_{0}$ to illustrate better the vectorization, the latter being a special case of the former). Then we have

$$
\mathbb{K}_{i, j}^{e}(K) \approx \frac{|K|}{d+1}\left(\left\langle\nabla \lambda_{\beta}, \mathbb{Q}^{n, l} \nabla \lambda_{\alpha}\right\rangle \lambda^{s}+\left\langle\nabla \lambda_{\beta}, \mathbb{S}^{n, l} \nabla \lambda_{\alpha}\right\rangle \mu^{s}\right),
$$

with $\lambda^{s}=\sum_{\gamma=1}^{d+1} \lambda\left(\mathrm{q}^{\gamma}\right)$ and $\mu^{s}=\sum_{\gamma=1}^{d+1} \mu\left(\mathrm{q}^{\gamma}\right)$. The previous formula may now be vectorized as shown in Algorithm 5.4. This algorithm is based on the vectorization of the computation of the terms $\left\langle\nabla \lambda_{\beta}, \mathbb{A} \nabla \lambda_{\alpha}\right\rangle$, which is carried out with the function dotMatVecG in Algorithm [5.5 for any $d$-by- $d$ matrix $\mathbb{A}$ independent of the $d$-simplices of the mesh. In this algorithm, $\mathbb{G}$ is the array 
of gradients defined in (4.1), $\alpha$ and $\beta$ are indices in $\{1, \ldots, d+1\}$, and $\boldsymbol{X}$ is a 1-by-n $\mathrm{n}_{\text {me }}$ array such that $\boldsymbol{X}(k)=\left\langle\nabla \lambda_{\beta}, \mathbb{A} \nabla \lambda_{\alpha}\right\rangle$ on $K=T_{k}$.
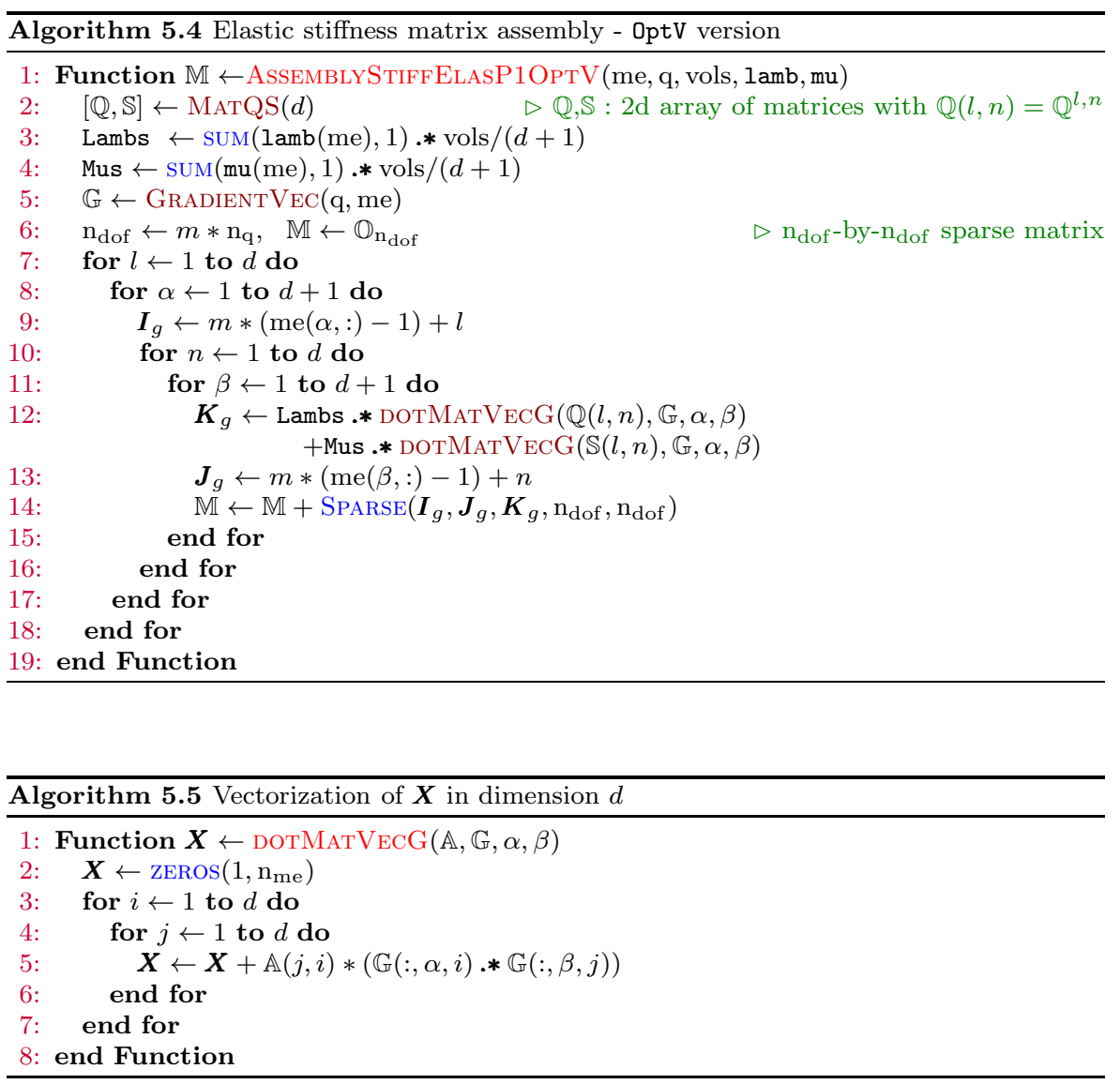

From Algorithm 5.4 it is straightforward to derive Algorithm [C.2 which uses the symmetry when the assembly matrix is symmetric.

We now present numerical results that illustrate the performance of the finite element assembly methods presented in this article.

\section{Benchmark results}

We consider the assembly of the stiffness and elastic stiffness matrices in $2 \mathrm{D}$ and $3 \mathrm{D}$, in the following vector languages

- Matlab (R2014b),

- Octave (3.8.1),

- Python 3.4.0 with NumPy[1.8.2] and SciPy[0.13.3]. 
We first compare the computation times of the different codes (base, OptV1, OptV2, OptV and OptVS), for each language considered. Then we compare OptVS code with a C implementation of the assembly using the SuiteSparse library 4.2.1 [10] ("CXSparse") and with FreeFEM++. A comparison of the performance of the OptVS code with recent and efficient Matlab/Octave codes is also given. In every benchmark the domain $\Omega$ is the unit disk in $2 \mathrm{D}$ and the unit sphere in $3 \mathrm{D}$. For each result we present the average computation time for at least five finite element assembly calculations.

6.1 Comparison of the base, OptV1, OptV2, OptV and OptVS assembly codes

We show in Figures 6.1 and 6.2 in logarithmic scales and for each vector language, the performance of the assembly codes versus the matrix dimension $\mathrm{n}_{\text {dof }}$, for the $2 \mathrm{D}$ stiffness and $3 \mathrm{D}$ elastic stiffness matrices respectively. We observe that the OptVS version is the fastest one and its complexity is $\mathcal{O}\left(\mathrm{n}_{\text {dof }}\right)$.
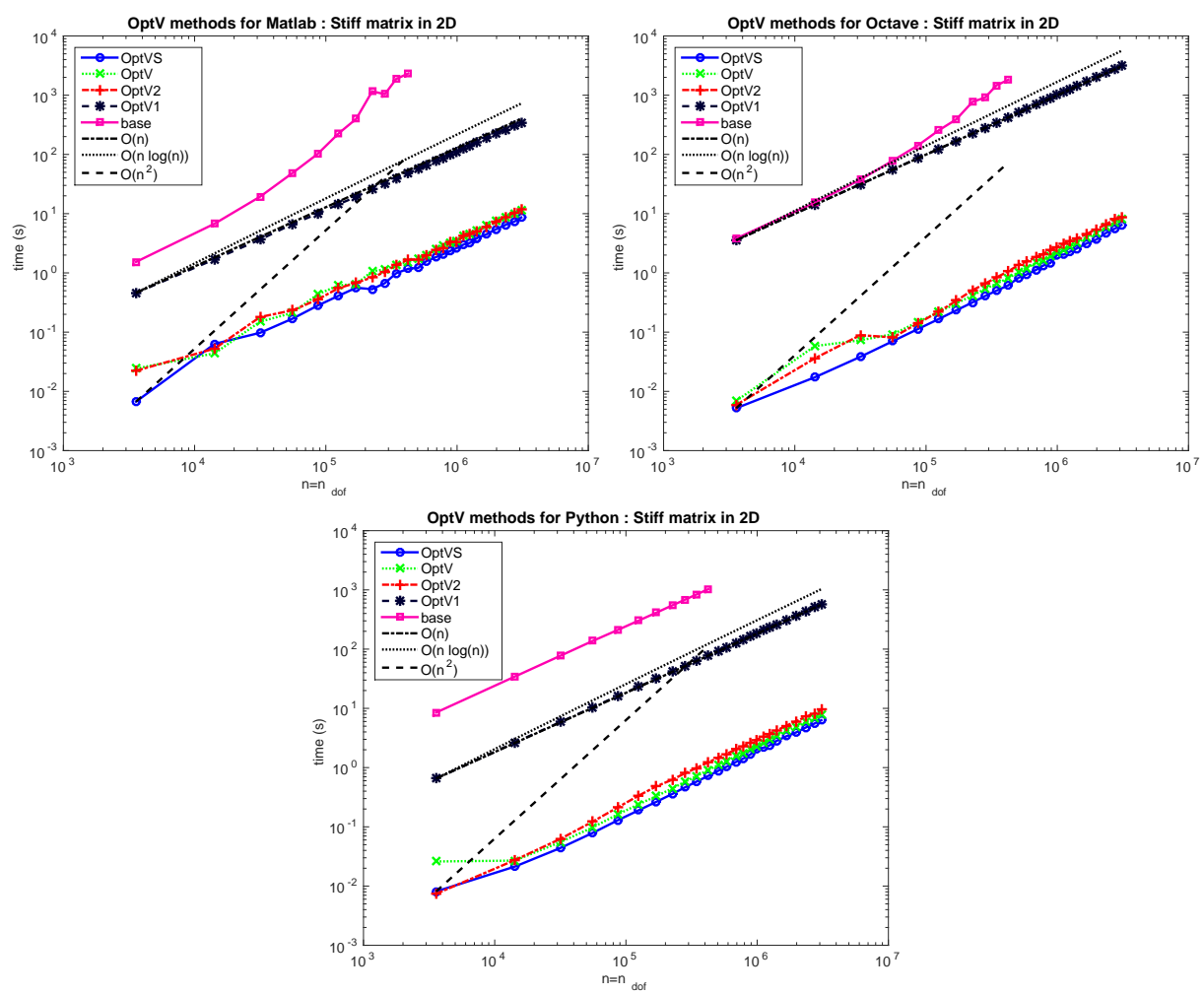

Fig. 6.1: Stiffness matrix (2D): comparison of base, OptV1, OptV2, OptV and OptVS codes in Matlab (top left), Octave (top right) and Python (bottom). 

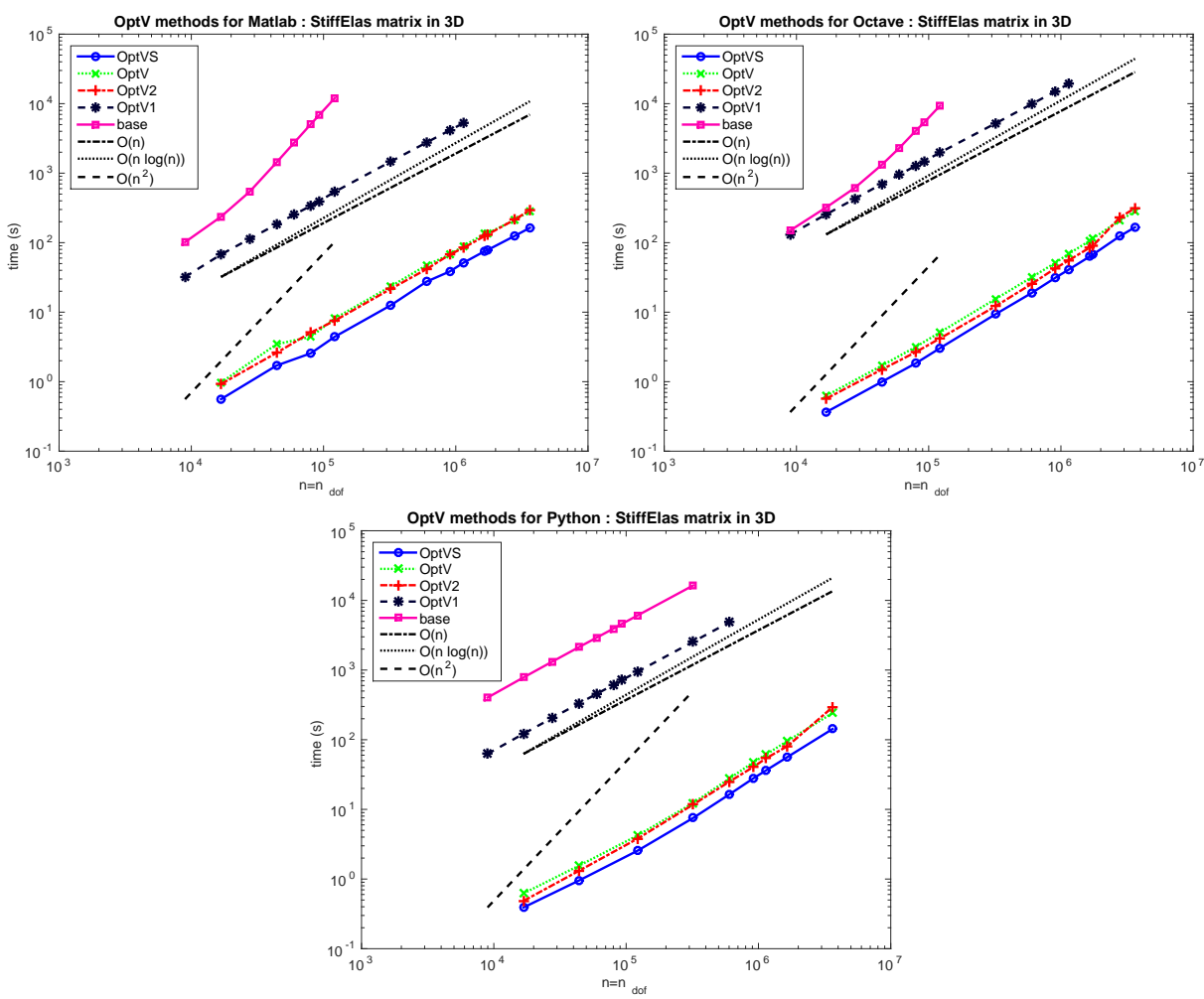

Fig. 6.2: Elastic stiffness matrix (3D): comparison of base, OptV1, OptV2, OptV and OptVS codes in Matlab (top left), Octave (top right) and Python (bottom).

For the stiffness matrix in 2D, the OptV1 version is about 40, 95 and 550 times slower in Matlab, Python and Octave respectively. Its numerical complexity is $\mathcal{O}\left(\mathrm{n}_{\text {dof }}\right)$. The complexity of the less performing method, the base version, is $\mathcal{O}\left(\mathrm{n}_{\text {dof }}^{2}\right)$ in Matlab and Octave, while it seems to be $\mathcal{O}\left(\mathrm{n}_{\text {dof }}\right)$ in Python. This is partly due to the use of the LIL format in the sparse matrix assembly in Python, the conversion to the CSC format being included in the computation time. We obtain similar results for the stiffness matrix in 3D and the elastic stiffness matrices in 2D and 3D. Computation times and OptVS speedup are given in Tables 6.1 and 6.2 for the 2D stiffness and 3D elastic stiffness matrices respectively. For the 3D stiffness and the $2 \mathrm{D}$ elastic stiffness matrices one can refer respectively to Tables A.3 and A.4. We observe that the performance differences of the stiffness and elastic stiffness matrix assemblies in $2 \mathrm{D}$ and $3 \mathrm{D}$ are partly due to the increase of the data: on the unit disk (2D) and the unit sphere (3D), we have $\mathrm{n}_{\mathrm{me}} \approx 2 \mathrm{n}_{\mathrm{q}}$ and $\mathrm{n}_{\mathrm{me}} \approx 6 \mathrm{n}_{\mathrm{q}}$ respectively. For matrices of the same size (i.e. for an equal $n_{\text {dof }}$ ), in comparison to the $2 \mathrm{D}$ stiffness matrix, the number of local values to be computed are 2, 4 and 16 times larger for the 2D elastic stiffness, the 3D stiffness and the 3D elastic stiffness matrices respectively. 


\begin{tabular}{|c|c|c|c|c|c|}
\hline \multicolumn{6}{|c|}{ StiffAssembling2DP1 - Matlab } \\
\hline$n_{\text {dof }}$ & OptVS & OptV & OptV2 & OptV1 & base \\
\hline 14222 & $\begin{array}{l}.063(\mathrm{~s}) \\
x_{1}\end{array}$ & $\begin{array}{l}.044(\mathrm{~s}) \\
\times \quad 692\end{array}$ & $\begin{array}{l}.053(\mathrm{~s}) \\
\times \quad 833\end{array}$ & $\begin{array}{l}1.70_{(\mathrm{s})} \\
\times 26.8\end{array}$ & $\begin{array}{l}6.79_{(\mathrm{s})} \\
\times 107\end{array}$ \\
\hline 125010 & $\begin{array}{c}411(\mathrm{~s}) \\
\times 1\end{array}$ & $\begin{array}{l}.617(s) \\
\times 1.5\end{array}$ & $\begin{array}{l}.553(s) \\
\times 1.35\end{array}$ & $\begin{array}{l}14.4(\mathrm{~s}) \\
\times \quad 35.1\end{array}$ & $\begin{array}{r}226(s) \\
\times 550\end{array}$ \\
\hline 343082 & $\begin{array}{c}.985(\mathrm{~s}) \\
\times 1\end{array}$ & $\begin{array}{r}1.37(\mathrm{~s}) \\
\times \quad 1.39\end{array}$ & $\begin{array}{r}1.36(\mathrm{~s}) \\
\times 1.38\end{array}$ & $\begin{array}{r}39.1(\mathrm{~s}) \\
\times \quad 39.7\end{array}$ & $\begin{array}{r}1873(\mathrm{~s}) \\
\times \quad 1902\end{array}$ \\
\hline 885521 & $\begin{array}{c}2.34(\mathrm{~s}) \\
\mathrm{x} 1\end{array}$ & $\begin{array}{l}3.24(\mathrm{~s}) \\
\times 1.39\end{array}$ & $\begin{array}{l}3.29(\mathrm{~s}) \\
\times 1.41\end{array}$ & $\begin{array}{r}99.7(\mathrm{~s}) \\
\times \quad 42.7\end{array}$ & - \\
\hline 1978602 & $\begin{array}{c}5.45(\mathrm{~s}) \\
\times 1\end{array}$ & $\begin{array}{r}7.60(\mathrm{~s}) \\
\times \quad 1.40\end{array}$ & $\begin{array}{r}7.28(\mathrm{~s}) \\
\times 1.34\end{array}$ & $\begin{array}{r}223(s) \\
\times \quad 40.9\end{array}$ & - \\
\hline
\end{tabular}

StiffAssembling2DP1 - Octave

\begin{tabular}{|c|c|c|c|c|c|}
\hline$n_{\text {dof }}$ & OptVS & OptV & OptV2 & OptV1 & base \\
\hline 14222 & $\begin{array}{c}.017(\mathrm{~s}) \\
\times 1\end{array}$ & $\begin{array}{l}.058(\mathrm{~s}) \\
\times \quad 3.36\end{array}$ & $\begin{array}{l}.036(\mathrm{~s}) \\
\times \quad 2.09 \\
\end{array}$ & $\begin{array}{c}14.3(\mathrm{~s}) \\
\times 826\end{array}$ & $\begin{array}{r}15.4(\mathrm{~s}) \\
\times 888\end{array}$ \\
\hline 125010 & $\begin{array}{c}.167(\mathrm{~s}) \\
\mathrm{x} 1\end{array}$ & $\begin{array}{r}.218(\mathrm{~s}) \\
\times \quad 1.31\end{array}$ & $\begin{array}{l}.221(\mathrm{~s}) \\
\times \quad 1.33\end{array}$ & $\begin{array}{r}124(s) \\
\times 742\end{array}$ & $\begin{array}{l}255(\mathrm{~s}) \\
\times 1533\end{array}$ \\
\hline 343082 & $\begin{array}{c}.499(\mathrm{~s}) \\
\times 1\end{array}$ & $\begin{array}{r}.656(\mathrm{~s}) \\
\times \quad 1.32\end{array}$ & $\begin{array}{r}835(\mathrm{~s}) \\
\times \quad 1.67\end{array}$ & $\begin{array}{c}340(\mathrm{~s}) \\
\mathrm{x} 681\end{array}$ & $\begin{array}{l}1458(\mathrm{~s}) \\
\times 2923 \\
\end{array}$ \\
\hline 885521 & $\begin{array}{c}1.47(\mathrm{~s}) \\
\times \quad 1\end{array}$ & $\begin{array}{rr}1.91(\mathrm{~s}) \\
\times \quad 1.30 \\
\end{array}$ & $\begin{array}{l}2.43(\mathrm{~s}) \\
\times \quad 1.65 \\
\end{array}$ & $\begin{array}{r}899(\mathrm{~s}) \\
\times 613 \\
\end{array}$ & - \\
\hline 1978602 & $\begin{array}{c}3.64(\mathrm{~s}) \\
\times 1\end{array}$ & $\begin{array}{l}4.63(\mathrm{~s}) \\
\times \quad 1.27 \\
\end{array}$ & $\begin{array}{r}5.44(\mathrm{~s}) \\
\times \quad 1.49 \\
\end{array}$ & $\begin{array}{c}2007(\mathrm{~s}) \\
\times 551 \\
\end{array}$ & - \\
\hline
\end{tabular}

StiffAssembling2DP1 - Python

\begin{tabular}{|c|c|c|c|c|c|}
\hline$n_{d o f}$ & OptVs & OptV & OptV2 & OptV1 & base \\
\hline 14222 & $\begin{array}{c}.021(\mathrm{~s}) \\
\mathrm{x} 1\end{array}$ & $\begin{array}{l}.027(\mathrm{~s}) \\
\times \quad 1.26\end{array}$ & $\begin{array}{l}.027(\mathrm{~s}) \\
\times \quad 1.29\end{array}$ & $\begin{array}{c}2.64(\mathrm{~s}) \\
\times \quad 124\end{array}$ & $\begin{array}{l}34.4(s) \\
\times 1614\end{array}$ \\
\hline 125010 & $\begin{array}{c}.190(\mathrm{~s}) \\
\times 1\end{array}$ & $\begin{array}{l}.241(\mathrm{~s}) \\
\times 1.26\end{array}$ & $\begin{array}{l}.336(\mathrm{~s}) \\
\times 1.77\end{array}$ & $\begin{array}{c}23.2(\mathrm{~s}) \\
\mathrm{x} \quad 122\end{array}$ & $\begin{array}{l}303(\mathrm{~s}) \\
\mathrm{x} \quad 1594\end{array}$ \\
\hline 343082 & $\begin{array}{c}.576(\mathrm{~s}) \\
\times 1\end{array}$ & $\begin{array}{l}.716(\mathrm{~s}) \\
\times 1.24 \\
\end{array}$ & $\begin{array}{r}.980(\mathrm{~s}) \\
\times \quad 1.70 \\
\end{array}$ & $\begin{array}{c}63.5(\mathrm{~s}) \\
\times 110 \\
\end{array}$ & $\begin{array}{r}833(\mathrm{~s}) \\
\times \quad 1445 \\
\end{array}$ \\
\hline 885521 & $\begin{array}{c}1.66(\mathrm{~s}) \\
\times 1\end{array}$ & $\begin{array}{l}2.05(\mathrm{~s}) \\
\times 1.23\end{array}$ & $\begin{array}{r}2.62(\mathrm{~s}) \\
\times 1.58\end{array}$ & $\begin{array}{r}164(\mathrm{~s}) \\
\times \quad 98.9\end{array}$ & - \\
\hline 1978602 & $\begin{array}{c}3.92(\mathrm{~s}) \\
\mathrm{x} 1\end{array}$ & $\begin{array}{r}4.85(\mathrm{~s}) \\
\times 1.24\end{array}$ & $\begin{array}{r}6.04(\mathrm{~s}) \\
\times 1.54\end{array}$ & $\begin{array}{r}368(\mathrm{~s}) \\
\times \quad 93.9\end{array}$ & - \\
\hline
\end{tabular}

Table 6.1: Stiffness matrix (2D) : comparison of OptVS, OptV, OptV1 and base codes in Matlab (top left), Octave (top right) and Python (bottom) giving time in seconds (top value) and OptVS speedup (bottom value).

\begin{tabular}{|c|c|c|c|c|c|}
\hline \multicolumn{6}{|c|}{ StiffElasAssembling3DP1 - Matlab } \\
\hline$n_{\text {dof }}$ & OptVS & OptV & \begin{tabular}{|l|l} 
OptV2 \\
\end{tabular} & OptV1 & base \\
\hline 16773 & $\begin{array}{c}.560(\mathrm{~s}) \\
\times 1 \\
\end{array}$ & $\begin{array}{r}.971(\mathrm{~s}) \\
\times \quad 1.73 \\
\end{array}$ & \begin{tabular}{|rr}
$.924(\mathrm{~s})$ \\
$\times \mathrm{x}$ \\
\end{tabular} & $\begin{array}{cc}67.6(\mathrm{~s}) \\
\times 121 \\
\end{array}$ & $\begin{array}{r}236(\mathrm{~s}) \\
\times 422 \\
\end{array}$ \\
\hline 44124 & $\begin{array}{c}1.70(\mathrm{~s}) \\
\times \quad 1 \\
\end{array}$ & $\begin{array}{r}3.45(s) \\
\times \quad 2.03 \\
\end{array}$ & $\begin{array}{rr}2.60(\mathrm{~s}) \\
\times \quad 1.52 \\
\end{array}$ & $\begin{array}{r}184(\mathrm{~s}) \\
\times \quad 108 \\
\end{array}$ & $\begin{array}{c}1427(\mathrm{~s}) \\
\times 837 \\
\end{array}$ \\
\hline 121710 & $\begin{array}{c}4.43(\mathrm{~s}) \\
\times \quad 1\end{array}$ & $\begin{array}{l}8.12(\mathrm{~s}) \\
\times 1.83\end{array}$ & \begin{tabular}{|l|}
$7.55(\mathrm{~s})$ \\
$\times 1.70$
\end{tabular} & $\begin{array}{r}540(\mathrm{~s}) \\
\times 122\end{array}$ & $\begin{array}{c}1 \mathrm{E}+4(\mathrm{~s}) \\
\times 2716\end{array}$ \\
\hline 601272 & $\begin{array}{c}27.5(s) \\
\times 1 \\
\end{array}$ & $\begin{array}{rr}47.4(\mathrm{~s}) \\
\times \quad 1.72 \\
\end{array}$ & \begin{tabular}{|l|l}
$41.5(\mathrm{~s})$ \\
$\mathrm{x} 1.51$ \\
\end{tabular} & $\begin{array}{c}2765(\mathrm{~s}) \\
\times \quad 101 \\
\end{array}$ & - \\
\hline 1144680 & $\begin{array}{c}51.5(\mathrm{~s}) \\
\times 1 \\
\end{array}$ & $\begin{array}{r}89.4(\mathrm{~s}) \\
\times \quad 1.74 \\
\end{array}$ & \begin{tabular}{|c|}
$84.2(\mathrm{~s})$ \\
$\mathrm{x} 1.64$ \\
\end{tabular} & $\begin{array}{c}5254(\mathrm{~s}) \\
\times 102 \\
\end{array}$ & - \\
\hline
\end{tabular}

\begin{tabular}{|c|c|c|c|c|c|}
\hline$n_{\text {dof }}$ & OptVs & $\mathrm{OptV}$ & OptV2 & OptV1 & base \\
\hline 16773 & $\begin{array}{c}.364(\mathrm{~s}) \\
\mathrm{x} 1\end{array}$ & $\begin{array}{l}.628(\mathrm{~s}) \\
\times 1.73\end{array}$ & $\begin{array}{l}.569(\mathrm{~s}) \\
\times 1.56\end{array}$ & $\begin{array}{r}255(\mathrm{~s}) \\
\times 701\end{array}$ & $\begin{array}{c}321(\mathrm{~s}) \\
\times 882\end{array}$ \\
\hline 44124 & $\begin{array}{c}.993(\mathrm{~s}) \\
\times 1\end{array}$ & $\begin{array}{l}1.69(\mathrm{~s}) \\
\times 1.71\end{array}$ & $\begin{array}{l}1.49(\mathrm{~s}) \\
\times 1.50\end{array}$ & $\begin{array}{r}698(\mathrm{~s}) \\
\times 703\end{array}$ & $\begin{array}{r}1314(\mathrm{~s}) \\
\times \quad 1323\end{array}$ \\
\hline 121710 & $\begin{array}{c}3.03(s) \\
\times \quad 1 \\
\end{array}$ & $\begin{array}{r}5.13(\mathrm{~s}) \\
\times \quad 1.69 \\
\end{array}$ & $\begin{array}{r}4.19(\mathrm{~s}) \\
\times \quad 1.38 \\
\end{array}$ & $\begin{array}{c}1976(\mathrm{~s}) \\
\times \quad 651 \\
\end{array}$ & $\begin{array}{r}9338(\mathrm{~s}) \\
\times \quad 3078 \\
\end{array}$ \\
\hline 601272 & $\begin{array}{c}18.9 \text { (s) } \\
\times 1\end{array}$ & $\begin{array}{l}31.7(\mathrm{~s}) \\
\times \quad 1.68\end{array}$ & $\begin{array}{r}25.5(\mathrm{~s}) \\
\times 1.35\end{array}$ & $\begin{array}{c}9853(\mathrm{~s}) \\
\text { x 521 }\end{array}$ & - \\
\hline 1144680 & $\begin{array}{c}40.9(\mathrm{~s}) \\
\times 1\end{array}$ & $\begin{array}{l}69.1(\mathrm{~s}) \\
\times \quad 1.69\end{array}$ & $\begin{array}{l}55.6(\mathrm{~s}) \\
\times 1.36\end{array}$ & $\begin{array}{c}2 \mathrm{E}+4(\mathrm{~s}) \\
\mathrm{x} 471\end{array}$ & - \\
\hline
\end{tabular}

\begin{tabular}{|c|c|c|c|c|c|}
\hline$n_{\text {dof }}$ & OptVS & OptV & OptV2 & OptV1 & base \\
\hline 16773 & $\begin{array}{c}.391(\mathrm{~s}) \\
\mathrm{x} 1\end{array}$ & $\begin{array}{l}.622(\mathrm{~s}) \\
\times \quad 1.59\end{array}$ & $\begin{array}{r}.486(\mathrm{~s}) \\
\times 1.24\end{array}$ & $\begin{array}{l}1222_{(\mathrm{s})} \\
\times 312\end{array}$ & $\begin{array}{r}784(\mathrm{~s}) \\
\times \quad 2004\end{array}$ \\
\hline 44124 & $\begin{array}{c}954(\mathrm{~s}) \\
\times 1 \\
\end{array}$ & $\begin{array}{r}1.56(\mathrm{~s}) \\
\times 1.63 \\
\end{array}$ & $\begin{array}{rr}1.32(\mathrm{~s}) \\
\times 1.38 \\
\end{array}$ & $\begin{array}{r}333(\mathrm{~s}) \\
\times 349 \\
\end{array}$ & $\begin{array}{r}2141(\mathrm{~s}) \\
\times 2243 \\
\end{array}$ \\
\hline 121710 & $\begin{array}{c}2.55(\mathrm{~s}) \\
\times 1 \\
\end{array}$ & $\begin{array}{r}4.21(\mathrm{~s}) \\
\times \quad 1.65 \\
\end{array}$ & $\begin{array}{rr}3.79(\mathrm{~s}) \\
\times \quad 1.49 \\
\end{array}$ & $\begin{array}{r}946(\mathrm{~s}) \\
\times \quad 372 \\
\end{array}$ & $\begin{array}{r}6071(\mathrm{~s}) \\
\times \quad 2384 \\
\end{array}$ \\
\hline 601272 & $\begin{array}{c}16.4 \text { (s) } \\
\times 1\end{array}$ & $\begin{array}{r}27.6(\mathrm{~s}) \\
\times 1.68\end{array}$ & $\begin{array}{l}24.9(\mathrm{~s}) \\
\times 1.52\end{array}$ & $\begin{array}{r}4850(\mathrm{~s}) \\
\times 296\end{array}$ & - \\
\hline 1144680 & $\begin{array}{c}36.4(\mathrm{~s}) \\
\times 1\end{array}$ & $\begin{array}{r}61.5(\mathrm{~s}) \\
\times 1.69 \\
\end{array}$ & $\begin{array}{r}54.2(\mathrm{~s}) \\
\times 1.49 \\
\end{array}$ & 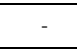 & - \\
\hline
\end{tabular}

Table 6.2: Elastic stiffness matrix (3D) : comparison of OptVS, OptV, OptV2, OptV1 and base codes in Matlab (top left), Octave (top right) and Python (bottom) giving time in seconds (top value) and OptVS speedup (bottom value). 
In Figure 6.3 we compare the maximum of memory for OptVS, OptV and OptV2 codes. The OptV2 method is more consuming than OptVS and OptV respectively by a factor between 5 and 6.3 and between 6 and 8.9 depending on the language.

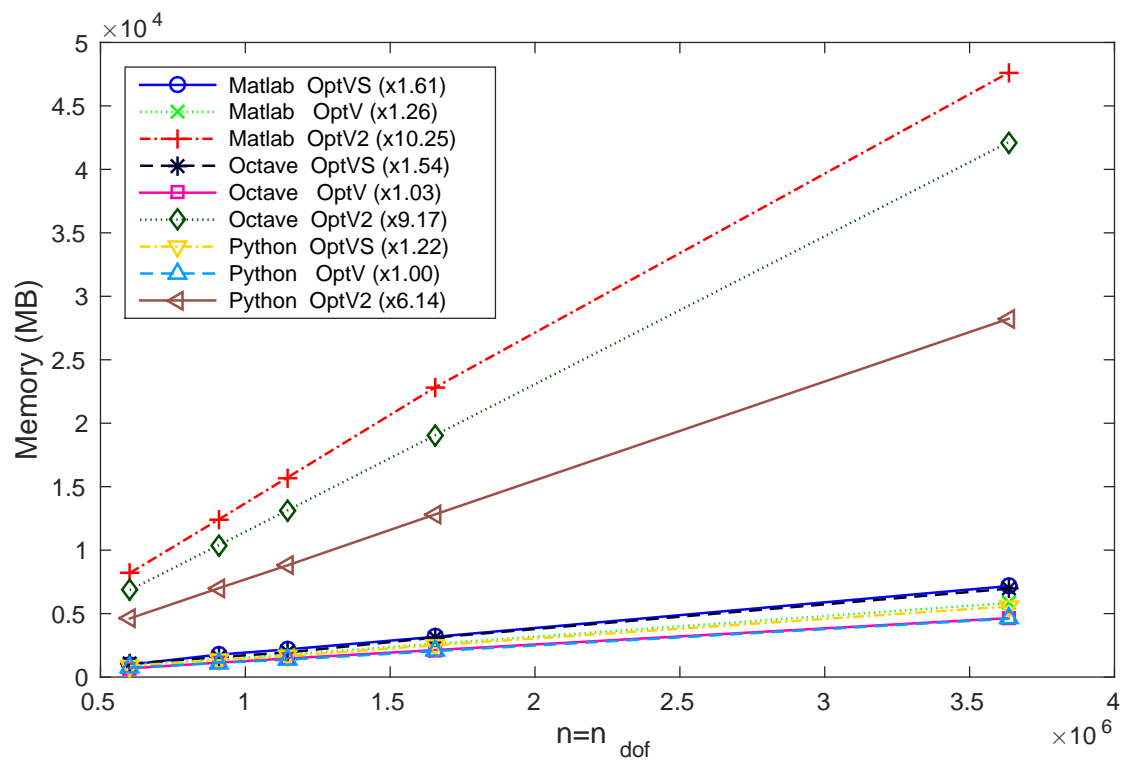

Fig. 6.3: Elastic stiffness matrix (3D): memory usage in MB and ratio between the slope of each method and OptV in Python (in the caption)

\subsection{Comparison of the OptVS version with CXSparse and FreeFEM++}

In Tables 6.3 the OptVS codes in Matlab/Octave/Python are compared with a $\mathrm{C}$ implementation of the assembly (OptV1 version) using the SuiteSparse library [10] ("CXSparse") and with a FreeFEM++ code for the stiffness matrix in $2 \mathrm{D}$ and the elastic stiffness matrix in $3 \mathrm{D}$.

The computation cost for the stiffness matrix in 3D and the elastic stiffness matrix in 2D are given in Tables A.1 and A.2. We observe that OptVS version is approximately 1.5 and 5.5 times in Matlab, 2 and 7.5 times in Octave, and 2.1 and 8.5 times in Python faster than FreeFEM++. Compared to C, computation times are multiplied by a factor between 2.5 and 4.7 in Matlab, 1.9 and 3.7 in Octave, and 1.8 and 3.2 in Python. Unlike what is commonly believed the performance is not radically worse than that of $\mathrm{C}$. 


\begin{tabular}{|c|c|c|c|c|c|}
\hline$n_{d o f}$ & $\begin{array}{c}\text { CXSparse } \\
(4.2 .1) \\
\end{array}$ & $\begin{array}{l}\text { Matlab } \\
(2014 b) \\
\end{array}$ & $\begin{array}{l}\text { Octave } \\
(3.8 .1) \\
\end{array}$ & $\begin{array}{l}\text { Python } \\
(3.4 .0) \\
\end{array}$ & $\begin{array}{c}\text { FreeFEM } \\
(3.31) \\
\end{array}$ \\
\hline 14222 & $\begin{array}{c}0.014(\mathrm{~s}) \\
\times 1.00\end{array}$ & $\begin{array}{c}0.063(\mathrm{~s}) \\
\times 4.64\end{array}$ & $\begin{array}{c}0.017(\mathrm{~s}) \\
\times \quad 1.26\end{array}$ & $\begin{array}{c}0.021(\mathrm{~s}) \\
\times 1.56\end{array}$ & $\begin{array}{c}0.071(\mathrm{~s}) \\
\mathrm{x} 5.16\end{array}$ \\
\hline 125010 & $\begin{array}{c}0.073(\mathrm{~s}) \\
\times \quad 1.00\end{array}$ & $\begin{array}{c}0.411(\mathrm{~s}) \\
\times 5.62\end{array}$ & $\begin{array}{c}0.167(\mathrm{~s}) \\
\times \quad 2.28\end{array}$ & $\begin{array}{c}0.190(\mathrm{~s}) \\
\times 2.60\end{array}$ & $\begin{array}{c}0.501(\mathrm{~s}) \\
\times \quad 6.85\end{array}$ \\
\hline 343082 & $\begin{array}{c}0.221(\mathrm{~s}) \\
\mathrm{x} 1.00\end{array}$ & $\begin{array}{c}0.985(\mathrm{~s}) \\
\times 4.46\end{array}$ & $\begin{array}{c}0.499(\mathrm{~s}) \\
\times \quad 2.26\end{array}$ & $\begin{array}{c}0.576(\mathrm{~s}) \\
\times 2.61\end{array}$ & $\begin{array}{c}1.421(\mathrm{~s}) \\
\times \quad 6.43\end{array}$ \\
\hline 885521 & $\begin{array}{c}0.606(\mathrm{~s}) \\
\times \quad 1.00\end{array}$ & $\begin{array}{c}2.337(\mathrm{~s}) \\
\mathrm{x} 3.86\end{array}$ & $\begin{array}{c}1.467(\mathrm{~s}) \\
\times 2.42\end{array}$ & $\begin{array}{c}1.660(\mathrm{~s}) \\
\times 2.74\end{array}$ & $\begin{array}{c}3.692(\mathrm{~s}) \\
\times \quad 6.10\end{array}$ \\
\hline 1978602 & $\begin{array}{c}1.354(\mathrm{~s}) \\
\times \quad 1.00\end{array}$ & $\begin{array}{c}5.446(\mathrm{~s}) \\
\times 4.02\end{array}$ & $\begin{array}{c}3.644(\mathrm{~s}) \\
\times \quad 2.69\end{array}$ & $\begin{array}{c}3.920(\mathrm{~s}) \\
\times \quad 2.89\end{array}$ & $\begin{array}{c}8.305(\mathrm{~s}) \\
\times \quad 6.13\end{array}$ \\
\hline
\end{tabular}

\begin{tabular}{|c||c|c|c|c|c|}
\hline$n_{\text {dof }}$ & $\begin{array}{c}\text { CXSparse } \\
(4.2 .1)\end{array}$ & $\begin{array}{c}\text { Matlab } \\
(2014 \mathrm{~b})\end{array}$ & $\begin{array}{c}\text { Octave } \\
(3.8 .1)\end{array}$ & $\begin{array}{c}\text { Python } \\
(3.4 .0)\end{array}$ & $\begin{array}{c}\text { FreeFEM } \\
(3.31)\end{array}$ \\
\hline \hline \multirow{2}{*}{16773} & $\begin{array}{c}0.137(\mathrm{~s}) \\
\mathrm{x} 1.00\end{array}$ & $\begin{array}{c}0.560(\mathrm{~s}) \\
\mathrm{x} 4.09\end{array}$ & $\begin{array}{c}0.364(\mathrm{~s}) \\
\mathrm{x} 2.66\end{array}$ & $\begin{array}{c}0.391(\mathrm{~s}) \\
\mathrm{x} \mathrm{2.86}\end{array}$ & $\begin{array}{c}3.827(\mathrm{~s}) \\
\mathrm{x} 27.95\end{array}$ \\
\hline \multirow{2}{*}{44124} & $\begin{array}{c}0.398(\mathrm{~s}) \\
\mathrm{x} 1.00\end{array}$ & $\begin{array}{c}1.705(\mathrm{~s}) \\
\mathrm{x} 4.29\end{array}$ & $\begin{array}{c}0.993(\mathrm{~s}) \\
\mathrm{x} 2.50\end{array}$ & $\begin{array}{c}0.954(\mathrm{~s}) \\
\mathrm{x} 2.40\end{array}$ & $\begin{array}{c}10.440(\mathrm{~s}) \\
\mathrm{x} 26.26\end{array}$ \\
\hline \multirow{2}{*}{121710} & $\begin{array}{c}1.193(\mathrm{~s}) \\
\mathrm{x} 1.00\end{array}$ & $\begin{array}{c}4.433(\mathrm{~s}) \\
\mathrm{x} 3.72\end{array}$ & $\begin{array}{c}3.034(\mathrm{~s}) \\
\mathrm{x} 2.54\end{array}$ & $\begin{array}{c}2.547(\mathrm{~s}) \\
\mathrm{x} 2.13\end{array}$ & $\begin{array}{c}29.914(\mathrm{~s}) \\
\mathrm{x} 25.08\end{array}$ \\
\hline \multirow{2}{*}{601272} & $\begin{array}{c}6.386(\mathrm{~s}) \\
\mathrm{x} 1.00\end{array}$ & $\begin{array}{c}27.482(\mathrm{~s}) \\
\mathrm{x} 4.30\end{array}$ & $\begin{array}{c}18.894(\mathrm{~s}) \\
\mathrm{x} 2.96\end{array}$ & $\begin{array}{c}16.359(\mathrm{~s}) \\
\mathrm{x} 2.56\end{array}$ & $\begin{array}{c}152.553(\mathrm{~s}) \\
\mathrm{x} 23.89\end{array}$ \\
\hline \multirow{2}{*}{1144680} & $\begin{array}{c}12.477(\mathrm{~s}) \\
\mathrm{x} 1.00\end{array}$ & $\begin{array}{c}51.465(\mathrm{~s}) \\
\mathrm{x} 4.12\end{array}$ & $\begin{array}{c}40.940(\mathrm{~s}) \\
\mathrm{x} 3.28\end{array}$ & $\begin{array}{c}36.392(\mathrm{~s}) \\
\mathrm{x} 2.92\end{array}$ & $\begin{array}{c}293.307(\mathrm{~s}) \\
\mathrm{x} 23.51\end{array}$ \\
\hline
\end{tabular}

Table 6.3: 2D Stiffness matrix (top table) and 3D elastic stiffness matrix (bottom table) : computational cost versus $n_{\text {dof }}$, with the OptVS Matlab/Octave/Python version ( $2^{\text {nd }} / 3^{\text {rd }} / 4^{\text {th }}$ columns), with CXSparse $\left(1^{\text {st }}\right.$ column) and FreeFEM $++\left(5^{\text {th }}\right.$ column $)$; time in seconds (top value) and CXSparse speedup (bottom value).

\subsection{Comparison with other matrix assemblies in Matlab and Octave}

In Matlab/Octave other efficient algorithms have been proposed recently in 2, 3. 17, 34. More precisely, in [17, a vectorization is proposed, based on the permutation of two local loops with the one through the elements. This technique allows to easily assemble different matrices, from a reference element by affine transformation and by using a numerical integration. In 34, the implementation is based on extending element operations on arrays into operations on arrays of matrices, calling them matrix-array operations. The array elements are matrices instead of scalars and the operations are defined by the rules of linear algebra. Thanks to these new tools and a quadrature formula, different matrices are computed without any loop. In [3, for the assembly of the stiffness matrix in $2 \mathrm{D}$ associated to $\mathbb{P}_{1}$ finite elements, L. Chen constructs vectorially the nine sparse matrices corresponding to the nine elements of the local stiffness matrix in 2D and adds them to obtain the global matrix. The restriction to $d=2$ or 3 of Algorithm 4.2 corresponds to the method in 3 . 
We compare these codes to OptVS for the assembly of the stiffness matrix in 2D. In Tables 6.4 and 6.5, using Matlab and Octave respectively, computation times versus the number of vertices are given for the different codes. OptVS speedup is between 1 and 2.5 in comparison with the other vectorized codes for sufficiently fine meshes.

\begin{tabular}{|c|c|c|c|c|c|}
\hline$n_{\text {dof }}$ & OptVs & Chen & iFEM & HanJun & RahVal \\
\hline \multirow{2}{*}{125010} & $0.411(\mathrm{~s})$ & $0.616(\mathrm{~s})$ & $0.693(\mathrm{~s})$ & $0.646(\mathrm{~s})$ & $0.664(\mathrm{~s})$ \\
\hline & $\mathrm{x} 1.00$ & $\mathrm{x} 1.50$ & $\mathrm{x} 1.69$ & $\mathrm{x} 1.57$ & $\mathrm{x} 1.61$ \\
\hline \multirow{2}{*}{343082} & $0.985(\mathrm{~s})$ & $1.464(\mathrm{~s})$ & $1.257(\mathrm{~s})$ & $1.989(\mathrm{~s})$ & $2.096(\mathrm{~s})$ \\
\hline & $\mathrm{x} 1.00$ & $\mathrm{x} 1.49$ & $\mathrm{x} 1.28$ & $\mathrm{x} 2.02$ & $\mathrm{x} 2.13$ \\
\hline \multirow{2}{*}{885521} & $2.337(\mathrm{~s})$ & $3.307(\mathrm{~s})$ & $2.966(\mathrm{~s})$ & $4.372(\mathrm{~s})$ & $4.721(\mathrm{~s})$ \\
\hline & $\mathrm{x} 1.00$ & $\mathrm{x} 1.41$ & $\mathrm{x} 1.27$ & $\mathrm{x} 1.87$ & $\mathrm{x} 2.02$ \\
\hline \multirow{2}{*}{1978602} & $5.446(\mathrm{~s})$ & $9.286(\mathrm{~s})$ & $7.221(\mathrm{~s})$ & $9.813(\mathrm{~s})$ & $9.123(\mathrm{~s})$ \\
\hline & $\mathrm{x} 1.00$ & $\mathrm{x} 1.71$ & $\mathrm{x} 1.33$ & $\mathrm{x} 1.80$ & $\mathrm{x} 1.68$ \\
\hline \multirow{2}{*}{3085628} & $8.644(\mathrm{~s})$ & $12.332(\mathrm{~s})$ & $11.444(\mathrm{~s})$ & $14.562(\mathrm{~s})$ & $14.841(\mathrm{~s})$ \\
\hline & $\mathrm{x} 1.00$ & $\mathrm{x} 1.43$ & $\mathrm{x} 1.32$ & x 1.68 & x 1.72 \\
\hline
\end{tabular}

Table 6.4: Stiffness matrix (2D): computational cost in Matlab (R2014b) versus $\mathrm{n}_{\mathrm{q}}$, with the OptVS version (column 2) and with the codes in [2,3,17,34 (columns 3-6) : time in seconds (top value) and speedup (bottom value). The speedup reference is OptVS version.

\begin{tabular}{|c|c|c|c|c|c|}
\hline$n_{d o f}$ & OptVs & Chen & iFEM & HanJun & RahVal \\
\hline 125010 & $\begin{array}{c}0.167(\mathrm{~s}) \\
\times 1.00\end{array}$ & $\begin{array}{c}0.305(\mathrm{~s}) \\
\times 1.83\end{array}$ & $\begin{array}{c}0.288(\mathrm{~s}) \\
\times 1.73\end{array}$ & $\begin{array}{c}0.417(\mathrm{~s}) \\
\times 2.50\end{array}$ & $\begin{array}{c}0.486(\mathrm{~s}) \\
\mathrm{x} 2.92\end{array}$ \\
\hline 343082 & $\begin{array}{c}0.499(\mathrm{~s}) \\
\times \quad 1.00\end{array}$ & $\begin{array}{c}0.823(\mathrm{~s}) \\
\times 1.65\end{array}$ & $\begin{array}{c}0.644(\mathrm{~s}) \\
\times \quad 1.29\end{array}$ & $\begin{array}{c}1.299(\mathrm{~s}) \\
\times 2.60\end{array}$ & $\begin{array}{c}1.245(\mathrm{~s}) \\
\times 2.50\end{array}$ \\
\hline 885521 & $\begin{array}{c}1.467(\mathrm{~s}) \\
\mathrm{x} 1.00\end{array}$ & $\begin{array}{c}2.123(\mathrm{~s}) \\
\times \quad 1.45\end{array}$ & $\begin{array}{c}1.663(\mathrm{~s}) \\
\mathrm{x} 1.13\end{array}$ & $\begin{array}{c}3.720(\mathrm{~s}) \\
\times 2.54\end{array}$ & $\begin{array}{c}3.221(\mathrm{~s}) \\
\times \quad 2.20\end{array}$ \\
\hline 1978602 & $\begin{array}{c}3.644(\mathrm{~s}) \\
\mathrm{x} 1.00\end{array}$ & $\begin{array}{c}4.674(\mathrm{~s}) \\
\times \quad 1.28\end{array}$ & $\begin{array}{c}3.832(\mathrm{~s}) \\
\mathrm{x} 1.05\end{array}$ & $\begin{array}{c}8.279(\mathrm{~s}) \\
\times \quad 2.27\end{array}$ & $\begin{array}{c}7.164(\mathrm{~s}) \\
\times 1.97\end{array}$ \\
\hline 3085628 & $\begin{array}{c}6.457(\mathrm{~s}) \\
\mathrm{x} 1.00\end{array}$ & $\begin{array}{c}7.786(\mathrm{~s}) \\
\mathrm{x} \quad 1.21\end{array}$ & $\begin{array}{c}6.642(\mathrm{~s}) \\
\mathrm{x} 1.03\end{array}$ & $\begin{array}{c}13.523(\mathrm{~s}) \\
\times \quad 2.09\end{array}$ & $\begin{array}{c}11.583(\mathrm{~s}) \\
\mathrm{x} 1.79\end{array}$ \\
\hline
\end{tabular}

Table 6.5: Stiffness matrix (2D): computational cost in Octave (3.8.1) versus $\mathrm{n}_{\mathrm{q}}$, with the OptVS version (column 2) and with the codes in [2,3, 17, 34 (columns 3-6) : time in seconds (top value) and speedup (bottom value). The speedup reference is OptVS version.

In Figure 6.4 we compare the memory costs in Matlab of our assembly codes with the other ones. As expected the consumption of OptVS and OptV methods are observed to be close to that of iFEM and lower than that of the other codes. 


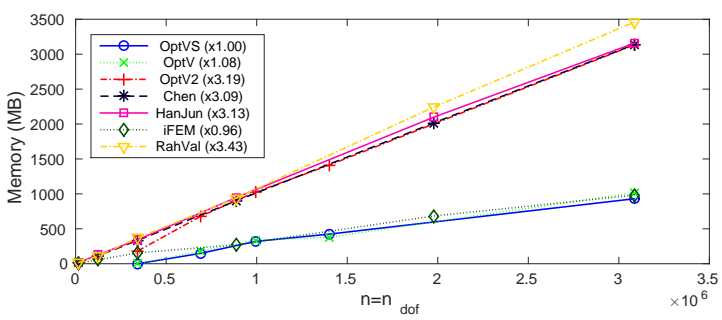

Fig. 6.4: Stiffness matrix (2D): memory usage in MB and ratio between the slope of each method and OptVS (in the caption)

\section{Conclusion and work in progress}

We presented vectorized algorithms for the assembly of $\mathbb{P}_{1}$ finite element matrices in arbitrary dimension. The implementation of these algorithms has been done in different vector languages such as Matlab, Octave and Python to calculate the stiffness and elastic stiffness matrices. Computation times of different versions (vectorized or not) have been compared in several interpreted languages and C. Numerical examples show the efficiency of the OptV2, OptV and OptVS algorithms. More precisely, for the OptVS method, the assembly of the stiffness matrix in $2 \mathrm{D}$ of size $10^{6}$ is performed in $2.6,1.75$ and 2 seconds with Matlab, Octave and Python respectively and in 0.75 seconds with C. Less performance is obtained for the assembly of the elastic stiffness matrix in 3D: a matrix of size $10^{6}$ is computed in $45,35.8$ and 31.8 seconds, with Matlab, Octave and Python respectively and in 10.9 seconds with C. Moreover we observed that OptVS is about 1.4 times faster than the non-symmetrized versions OptV and OptV2. OptV and OptVS methods are less memory consuming than OptV2. Preliminary results towards the extension to $\mathbb{P}_{k}$ finite elements are given in the Appendix. The algorithms in arbitrary dimension for piecewise polynomials of higher order, is the subject of a future paper. The OptV2 algorithm has been also implemented with a NVIDIA GPU3, using the Thrust and Cusp libraries. For the 2D elastic stiffness and 3D stiffness matrices, the OptV2 code is respectively 3.5 and 7 times faster on GPU than the $\mathrm{C}$ code (the time for GPU/CPU data and matrix transfers is taken into account).

Vectorization gave good performance and the vectorized code can be used for other matrices or discretizations, the only part of the code that have to be reviewed (which is probably the most difficult part) is the vectorization of the element matrix computation. We have seen that it is possible to efficiently assemble matrices of large size in interpreted languages. In this framework Python showed some very good performance even though Octave seems to be more efficient in some cases. Moreover the performance of our vectorized codes was better in Octave than in Matlab. The Python and Matlab/Octave codes are available online (see [7]).

3 GeForce GTX Titan Black, 2880 CUDA Core, 6Go Memory 


\section{A Additional benchmark results}

In this section, we consider the assembly of the 3D stiffness and 2D elastic stiffness matrices. In Tables A.1 and A.2 we compare the OptVS versions in Matlab/Octave/Python with a C implementation of the assembly (OptV1 version) using the SuiteSparse library [10] "CXSparse"), and with a FreeFEM++ version. In Tables A.3 and A.4 the computation times of OptVS, OptV, OptV2, OptV1 and base versions are compared in Matlab, Octave and Python. We observe similar results as in Section 6

\begin{tabular}{|c|c|c|c|c|c|}
\hline$n_{d o f}$ & $\begin{array}{c}\text { CXSparse } \\
(4.2 .1)\end{array}$ & $\begin{array}{l}\text { Matlab } \\
\text { (2014b) }\end{array}$ & $\begin{array}{l}\text { Octave } \\
(3.8 .1)\end{array}$ & $\begin{array}{c}\text { Python } \\
(3.4 .0)\end{array}$ & $\begin{array}{c}\text { FreeFEM } \\
(3.31)\end{array}$ \\
\hline 14708 & $\begin{array}{c}0.087(\mathrm{~s}) \\
\mathrm{x} \quad 1.00\end{array}$ & $\begin{array}{c}0.135(\mathrm{~s}) \\
\times \quad 1.55\end{array}$ & $\begin{array}{c}0.143(\mathrm{~s}) \\
\times \quad 1.64\end{array}$ & $\begin{array}{c}0.191(\mathrm{~s}) \\
\times \quad 2.19\end{array}$ & $\begin{array}{c}0.545(\mathrm{~s}) \\
\times \quad 6.26\end{array}$ \\
\hline 40570 & $\begin{array}{c}0.155(\mathrm{~s}) \\
\times 1.00\end{array}$ & $\begin{array}{c}0.461(\mathrm{~s}) \\
\times \quad 2.97\end{array}$ & $\begin{array}{c}0.293(\mathrm{~s}) \\
\times \quad 1.89\end{array}$ & $\begin{array}{c}0.377(\mathrm{~s}) \\
\times \quad 2.43\end{array}$ & $\begin{array}{r}1.571(\mathrm{~s}) \\
\times \quad 10.12\end{array}$ \\
\hline 200424 & $\begin{array}{c}1.014(\mathrm{~s}) \\
\mathrm{x} 1.00\end{array}$ & $\begin{array}{c}3.457(\mathrm{~s}) \\
\times \quad 3.41\end{array}$ & $\begin{array}{c}1.911(\mathrm{~s}) \\
\times 1.88\end{array}$ & $\begin{array}{c}1.935(\mathrm{~s}) \\
\times \quad 1.91\end{array}$ & $\begin{array}{c}8.742(\mathrm{~s}) \\
\times 8.62\end{array}$ \\
\hline 580975 & $\begin{array}{c}3.844(\mathrm{~s}) \\
\mathrm{x} 1.00\end{array}$ & $\begin{array}{c}9.767(\mathrm{~s}) \\
\times \quad 2.54\end{array}$ & $\begin{array}{c}7.193(\mathrm{~s}) \\
\times \quad 1.87\end{array}$ & $\begin{array}{c}6.804(\mathrm{~s}) \\
\times \quad 1.77\end{array}$ & $\begin{array}{c}26.970(\mathrm{~s}) \\
\times 7.02\end{array}$ \\
\hline 1747861 & $\begin{array}{c}10.752(\mathrm{~s}) \\
\times 1.00\end{array}$ & $\begin{array}{c}31.203(\mathrm{~s}) \\
\times 2.90\end{array}$ & $\begin{array}{c}31.008(\mathrm{~s}) \\
\times 2.88\end{array}$ & $\begin{array}{c}26.069(\mathrm{~s}) \\
\times 2.42\end{array}$ & $\begin{array}{c}84.698(\mathrm{~s}) \\
\times 7.88\end{array}$ \\
\hline
\end{tabular}

Table A.1: Stiffness matrix (3D) : computational cost versus $n_{\text {dof }}$, with the Optvs Matlab/Octave/Python version $\left(2^{\text {nd }} / 3^{\text {rd }} / 4^{\text {th }}\right.$ columns $)$, with CXSparse $\left(1^{\text {st }}\right.$ column $)$ and FreeFEM++ $\left(5^{\text {th }}\right.$ column $)$ : time in seconds (top value) and speedup (bottom value). The speedup reference is CXSparse code.

\begin{tabular}{|c|c|c|c|c|c|}
\hline$n_{d o f}$ & $\begin{array}{c}\text { CXSparse } \\
(4.2 .1)\end{array}$ & $\begin{array}{l}\text { Matlab } \\
(2014 b)\end{array}$ & $\begin{array}{l}\text { Octave } \\
(3.8 .1)\end{array}$ & $\begin{array}{c}\text { Python } \\
(3.4 .0)\end{array}$ & $\begin{array}{c}\text { FreeFEM } \\
(3.31) \\
\end{array}$ \\
\hline 28444 & $\begin{array}{c}0.023(\mathrm{~s}) \\
\times \quad 1.00\end{array}$ & $\begin{array}{c}0.139(\mathrm{~s}) \\
\times \quad 5.92\end{array}$ & $\begin{array}{c}0.115(\mathrm{~s}) \\
\times 4.88\end{array}$ & $\begin{array}{c}0.076(\mathrm{~s}) \\
\times \quad 3.25\end{array}$ & $\begin{array}{r}0.762(\mathrm{~s}) \\
\times \quad 32.47\end{array}$ \\
\hline 111838 & $\begin{array}{c}0.107(\mathrm{~s}) \\
\mathrm{x} 1.00\end{array}$ & $\begin{array}{c}0.412(\mathrm{~s}) \\
\times \quad 3.84\end{array}$ & $\begin{array}{c}0.321(\mathrm{~s}) \\
\times 2.99\end{array}$ & $\begin{array}{c}0.288(\mathrm{~s}) \\
\times \quad 2.69\end{array}$ & $\begin{array}{c}2.865(\mathrm{~s}) \\
\times \quad 26.75\end{array}$ \\
\hline 250020 & $\begin{array}{c}0.267(\mathrm{~s}) \\
\mathrm{x} 1.00\end{array}$ & $\begin{array}{c}1.046(\mathrm{~s}) \\
\times \quad 3.92\end{array}$ & $\begin{array}{c}0.727(\mathrm{~s}) \\
\times 2.73\end{array}$ & $\begin{array}{c}0.662(\mathrm{~s}) \\
\times \quad 2.48\end{array}$ & $\begin{array}{c}6.432(\mathrm{~s}) \\
\times \quad 24.11\end{array}$ \\
\hline 1013412 & $\begin{array}{c}1.133(\mathrm{~s}) \\
\mathrm{x} 1.00\end{array}$ & $\begin{array}{c}5.000(\mathrm{~s}) \\
\times \quad 4.41\end{array}$ & $\begin{array}{c}4.238(\mathrm{~s}) \\
\times \quad 3.74\end{array}$ & $\begin{array}{c}3.377(\mathrm{~s}) \\
\times \quad 2.98\end{array}$ & $\begin{array}{c}26.301(\mathrm{~s}) \\
\mathrm{x} 23.20\end{array}$ \\
\hline 2802258 & $\begin{array}{c}3.142(\mathrm{~s}) \\
\mathrm{x} 1.00\end{array}$ & $\begin{array}{c}14.867(\mathrm{~s}) \\
\times 4.73\end{array}$ & $\begin{array}{c}11.483(\mathrm{~s}) \\
\times \quad 3.65\end{array}$ & $\begin{array}{c}10.036(\mathrm{~s}) \\
\times \quad 3.19\end{array}$ & $\begin{array}{c}72.561(\mathrm{~s}) \\
\times \quad 23.10\end{array}$ \\
\hline
\end{tabular}

Table A.2: Elastic stiffness matrix (2D) : computational cost versus $n_{d o f}$, with the OptVs Matlab/Octave/Python version $\left(2^{\text {nd }} / 3^{\text {rd }} / 4^{\text {th }}\right.$ columns $)$, with CXSparse $\left(1^{\text {st }}\right.$ column $)$ and FreeFEM $++\left(5^{\text {th }}\right.$ column $):$ time in seconds (top value) and speedup (bottom value). The speedup reference is CXSparse code. 


\begin{tabular}{|c|c|c|c|c|c|}
\hline \multicolumn{6}{|c|}{ StiffAssembling3DP1 - Matlab } \\
\hline$n_{\text {dof }}$ & OptVS & OptV & OptV2 & OptV1 & base \\
\hline 14708 & $\begin{array}{c}135(\mathrm{~s}) \\
\mathrm{x} 1\end{array}$ & $\begin{array}{r}.409(\mathrm{~s}) \\
\times 3.03\end{array}$ & $\begin{array}{l}.442(s) \\
\times \quad 3.27\end{array}$ & $\begin{array}{l}5.07(\mathrm{~s}) \\
\times \quad 37.5\end{array}$ & $\begin{array}{c}25.6(\mathrm{~s}) \\
\mathrm{x} 189\end{array}$ \\
\hline 40570 & $\begin{array}{c}.461(\mathrm{~s}) \\
\times 1\end{array}$ & $\begin{array}{r}.827(\mathrm{~s}) \\
\times \quad 1.80\end{array}$ & $\begin{array}{r}.775(\mathrm{~s}) \\
\times \quad 1.68\end{array}$ & $\begin{array}{l}14.0(\mathrm{~s}) \\
\times 30.3\end{array}$ & $\begin{array}{l}112(s) \\
\times \quad 244\end{array}$ \\
\hline 200424 & $\begin{array}{c}3.46(\mathrm{~s}) \\
\times 1\end{array}$ & $\begin{array}{l}.69(\mathrm{~s}) \\
\times 1.36\end{array}$ & $\begin{array}{r}4.74(\mathrm{~s}) \\
\times 1.37\end{array}$ & $\begin{array}{l}69.6(\mathrm{~s}) \\
\times \quad 20.1\end{array}$ & $\begin{array}{c}3255(\mathrm{~s}) \\
\times 942\end{array}$ \\
\hline 580975 & $\begin{array}{c}9.77(\mathrm{~s}) \\
\times 1\end{array}$ & $\begin{array}{r}13.2(\mathrm{~s}) \\
\times \quad 1.35\end{array}$ & $\begin{array}{l}14.1(\mathrm{~s}) \\
\times \quad 1.44\end{array}$ & $\begin{array}{r}204(\mathrm{~s}) \\
\times \quad 20.9\end{array}$ & 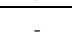 \\
\hline 1747861 & $\begin{array}{c}31.2(\mathrm{~s}) \\
\times 1\end{array}$ & $\begin{array}{r}40.4^{(\mathrm{s})} \\
\times \quad 1.29\end{array}$ & $\begin{array}{l}44.5(\mathrm{~s}) \\
\times \quad 1.42\end{array}$ & $\begin{array}{r}623(\mathrm{~s}) \\
\times \quad 20.0\end{array}$ & - \\
\hline
\end{tabular}

StiffAssembling3DP1 - Octave

\begin{tabular}{|c|c|c|c|c|c|}
\hline$n_{\text {dof }}$ & OptVS & OptV & OptV2 & OptV1 & base \\
\hline 14708 & $\begin{array}{c}143(\mathrm{~s}) \\
\mathrm{x} 1\end{array}$ & $\begin{array}{l}.177(\mathrm{~s}) \\
\times \quad 1.24 \\
\end{array}$ & $\begin{array}{l}146(\mathrm{~s}) \\
\times 1.02\end{array}$ & $\begin{array}{c}76.0(\mathrm{~s}) \\
\times 531\end{array}$ & $\begin{array}{c}77.3(\mathrm{~s}) \\
\mathrm{x} 540 \\
\end{array}$ \\
\hline 40570 & $\begin{array}{c}293(s) \\
\text { x } 1\end{array}$ & $\begin{array}{l}.446(\mathrm{~s}) \\
\times \quad 1.52 \\
\end{array}$ & $\begin{array}{r}.488(\mathrm{~s}) \\
\times 1.66 \\
\end{array}$ & $\begin{array}{c}216(\mathrm{~s}) \\
\times 737\end{array}$ & $\begin{array}{c}248 \text { (s) } \\
\times 844 \\
\end{array}$ \\
\hline 200424 & $\begin{array}{c}1.91(\mathrm{~s}) \\
\mathrm{x} 1\end{array}$ & $\begin{array}{l}2.37(\mathrm{~s}) \\
\times \quad 1.24 \\
\end{array}$ & $\begin{array}{l}3.15(\mathrm{~s}) \\
\times 1.65\end{array}$ & $\begin{array}{c}1120(\mathrm{~s}) \\
\times 586\end{array}$ & $\begin{array}{l}3041(\mathrm{~s}) \\
\times \quad 1592 \\
\end{array}$ \\
\hline 580975 & $\begin{array}{c}7.19(\mathrm{~s}) \\
\times 1\end{array}$ & $\begin{array}{l}9.15(\mathrm{~s}) \\
\times \quad 1.27 \\
\end{array}$ & $\begin{array}{l}10.6(\mathrm{~s}) \\
\times \quad 1.47 \\
\end{array}$ & $\begin{array}{c}3264(\mathrm{~s}) \\
\times \quad 454 \\
\end{array}$ & - \\
\hline 1747861 & $\begin{array}{c}31.0(\mathrm{~s}) \\
\times 1\end{array}$ & $\begin{array}{r}38.0(\mathrm{~s}) \\
\times \quad 1.22 \\
\end{array}$ & $\begin{array}{l}40.5(\mathrm{~s}) \\
\times \quad 1.31 \\
\end{array}$ & - & - \\
\hline
\end{tabular}

StiffAssembling3DP1 - Python

\begin{tabular}{|c|c|c|c|c|c|}
\hline$\overline{n_{d o f}}$ & OptVs & OptV & OptV2 & OptV1 & base \\
\hline 14708 & $\begin{array}{c}191(\mathrm{~s}) \\
\times 1\end{array}$ & $\begin{array}{l}.207(\mathrm{~s}) \\
\times \quad 1.08\end{array}$ & $\begin{array}{l}.258(\mathrm{~s}) \\
\times \quad 1.35\end{array}$ & $\begin{array}{l}12.8(\mathrm{~s}) \\
\times \quad 67.0\end{array}$ & $\begin{array}{l}172(\mathrm{~s}) \\
\times 903\end{array}$ \\
\hline 40570 & $\begin{array}{c}.377(\mathrm{~s}) \\
\times 1\end{array}$ & $\begin{array}{l}.530(\mathrm{~s}) \\
\times 1.41\end{array}$ & $\begin{array}{l}.823(s) \\
\times 2.19\end{array}$ & $\begin{array}{l}36.0(\mathrm{~s}) \\
\times 95.5\end{array}$ & $\begin{array}{r}488(s) \\
\times \quad 1295\end{array}$ \\
\hline 200424 & $\begin{array}{c}1.93(\mathrm{~s}) \\
\times 1\end{array}$ & $\begin{array}{r}2.50(\mathrm{~s}) \\
\times \quad 1.29\end{array}$ & $\begin{array}{r}4.15(\mathrm{~s}) \\
\times 2.14\end{array}$ & $\begin{array}{r}182(\mathrm{~s}) \\
\times \quad 94.0\end{array}$ & $\begin{array}{r}2480(s) \\
\times \quad 1282\end{array}$ \\
\hline 580975 & $\begin{array}{c}6.80(\mathrm{~s}) \\
\times 1\end{array}$ & $\begin{array}{l}8.89(\mathrm{~s}) \\
\times 1.31\end{array}$ & $\begin{array}{l}12.4(\mathrm{~s}) \\
\times 1.83\end{array}$ & $\begin{array}{l}541(\mathrm{~s}) \\
\times 79.5\end{array}$ & - \\
\hline 1747861 & $\begin{array}{c}26.1(\mathrm{~s}) \\
\times 1\end{array}$ & $\begin{array}{l}34.2(\mathrm{~s}) \\
\times 1.31\end{array}$ & $\begin{array}{l}40.3(\mathrm{~s}) \\
\times 1.55\end{array}$ & - & - \\
\hline
\end{tabular}

Table A.3: Stiffness matrix (3D) : comparison of OptVS, OptV, OptV2, OptV1 and base codes in Matlab (top left), Octave (top right) and Python (bottom) giving time in seconds (top value) and OptVS speedup (bottom value).

\begin{tabular}{|c|c|c|c|c|c|}
\hline \multicolumn{6}{|c|}{ StiffElasAssembling2DP1 - Matlab } \\
\hline$n_{d o f}$ & OptVS & OptV & OptV2 & OptV1 & base \\
\hline 28444 & $\begin{array}{c}.114(\mathrm{~s}) \\
\mathrm{x} 1 \\
\end{array}$ & $\begin{array}{r}.272(s) \\
\times \quad 2.39 \\
\end{array}$ & $\begin{array}{r}.185(\mathrm{~s}) \\
\times \quad 1.63 \\
\end{array}$ & $\begin{array}{c}16.9(\mathrm{~s}) \\
\times 148 \\
\end{array}$ & $\begin{array}{c}67.8(\mathrm{~s}) \\
\times 594 \\
\end{array}$ \\
\hline 250020 & $\begin{array}{c}1.01(\mathrm{~s}) \\
\mathrm{x} 1\end{array}$ & $\begin{array}{l}1.68(\mathrm{~s}) \\
\times \quad 1.65 \\
\end{array}$ & $\begin{array}{rr}1.91(\mathrm{~s}) \\
\times \quad 1.88 \\
\end{array}$ & $\begin{array}{r}150(\mathrm{~s}) \\
\times 148 \\
\end{array}$ & $\begin{array}{r}6156(\mathrm{~s}) \\
\times 6073 \\
\end{array}$ \\
\hline 686164 & $\begin{array}{c}3.20(\mathrm{~s}) \\
\mathrm{x} 1\end{array}$ & $\begin{array}{l}5.28(\mathrm{~s}) \\
\times 1.65 \\
\end{array}$ & $\begin{array}{l}5.42(\mathrm{~s}) \\
\times 1.70\end{array}$ & $\begin{array}{c}14(\mathrm{~s}) \\
\times \quad 129\end{array}$ & 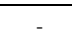 \\
\hline 1771042 & $\begin{array}{c}8.97(s) \\
\times 1\end{array}$ & $\begin{array}{l}14.7(\mathrm{~s}) \\
\times 1.64\end{array}$ & $\begin{array}{r}15.2(\mathrm{~s}) \\
\times 1.69\end{array}$ & $\begin{array}{c}1090(s) \\
\times \quad 122\end{array}$ & - \\
\hline 3957204 & $\begin{array}{c}21.6(\mathrm{~s}) \\
\times 1\end{array}$ & $\begin{array}{r}34.1(\mathrm{~s}) \\
\times \quad 1.58 \\
\end{array}$ & $\begin{array}{l}33.1(\mathrm{~s}) \\
\times 1.53\end{array}$ & - & - \\
\hline
\end{tabular}

\begin{tabular}{|c|c|c|c|c|c|}
\hline$n_{\text {dof }}$ & OptVS & OptV & OptV2 & OptV1 & base \\
\hline 28444 & $\begin{array}{c}.082(\mathrm{~s}) \\
\mathrm{x} 1\end{array}$ & $\begin{array}{l}129(\mathrm{~s}) \\
\times 1.57\end{array}$ & $\begin{array}{l}.091(\mathrm{~s}) \\
\times 1.11\end{array}$ & $\begin{array}{c}63.7(\mathrm{~s}) \\
\times 777\end{array}$ & $\begin{array}{l}88.6(\mathrm{~s}) \\
\times \quad 1080\end{array}$ \\
\hline 250020 & $\begin{array}{c}.726(\mathrm{~s}) \\
\mathrm{x} 1\end{array}$ & $\begin{array}{r}1.26(\mathrm{~s}) \\
\times \quad 1.74\end{array}$ & $\begin{array}{r}.915(\mathrm{~s}) \\
\times \quad 1.26\end{array}$ & $\begin{array}{r}564(\mathrm{~s}) \\
\times 777\end{array}$ & $\begin{array}{r}4485(s) \\
\times \quad 6176\end{array}$ \\
\hline 686164 & $\begin{array}{c}2.21(\mathrm{~s}) \\
\mathrm{x} 1\end{array}$ & $\begin{array}{l}3.57(\mathrm{~s}) \\
\times \quad 1.61\end{array}$ & $\begin{array}{l}3.36(\mathrm{~s}) \\
\times \quad 1.52\end{array}$ & $\begin{array}{c}1550(\mathrm{~s}) \\
\times 701\end{array}$ & - \\
\hline 1771042 & $\begin{array}{c}6.85(\mathrm{~s}) \\
\times 1\end{array}$ & $\begin{array}{l}10.7(\mathrm{~s}) \\
\times \quad 1.56\end{array}$ & $\begin{array}{l}9.39(\mathrm{~s}) \\
\times 1.37\end{array}$ & - & - \\
\hline 3957204 & $\begin{array}{c}16.2(\mathrm{~s}) \\
\times \quad 1\end{array}$ & $\begin{array}{r}25.8(\mathrm{~s}) \\
\times \quad 1.59\end{array}$ & $\begin{array}{r}22.0(\mathrm{~s}) \\
\times \quad 1.36\end{array}$ & - & - \\
\hline
\end{tabular}

StiffElas Assembling2DP1 - Python

\begin{tabular}{|c|c|c|c|c|c|}
\hline$n_{\text {dof }}$ & OptVS & OptV & OptV2 & OptV1 & base \\
\hline 28444 & $\begin{array}{c}.136(\mathrm{~s}) \\
\times 1 \\
\end{array}$ & $\begin{array}{r}.207(\mathrm{~s}) \\
\times \quad 1.53 \\
\end{array}$ & $\begin{array}{r}.202(\mathrm{~s}) \\
\times \quad 1.49 \\
\end{array}$ & $\begin{array}{c}30.0(s) \\
\times \quad 221 \\
\end{array}$ & $\begin{array}{r}183(\mathrm{~s}) \\
\times \quad 1348 \\
\end{array}$ \\
\hline 250020 & $\begin{array}{c}.721(\mathrm{~s}) \\
\mathrm{x} 1\end{array}$ & $\begin{array}{l}1.08(\mathrm{~s}) \\
\times \quad 1.50\end{array}$ & $\begin{array}{c}1.22(\mathrm{~s}) \\
\times 1.7\end{array}$ & $\begin{array}{r}277(\mathrm{~s}) \\
\times 384\end{array}$ & $\begin{array}{l}1639(\mathrm{~s}) \\
\times \quad 2274\end{array}$ \\
\hline 686164 & $\begin{array}{c}2.05(\mathrm{~s}) \\
\mathrm{x} 1 \\
\end{array}$ & $\begin{array}{r}3.06(\mathrm{~s}) \\
\times \quad 1.50 \\
\end{array}$ & $\begin{array}{rr}3.55(\mathrm{~s}) \\
\times \quad 1.73 \\
\end{array}$ & $\begin{array}{r}761(\mathrm{~s}) \\
\times \quad 372 \\
\end{array}$ & $\begin{array}{ll}- & - \\
\end{array}$ \\
\hline 1771042 & $\begin{array}{c}6.06(\mathrm{~s}) \\
\times 1 \\
\end{array}$ & $\begin{array}{r}9.12(\mathrm{~s}) \\
\times \quad 1.50 \\
\end{array}$ & $\begin{array}{r}9.58(\mathrm{~s}) \\
\times \quad 1.58 \\
\end{array}$ & $\begin{array}{l}- \\
-\end{array}$ & - \\
\hline 3957204 & $\begin{array}{c}14.0(\mathrm{~s}) \\
\mathrm{x} 1\end{array}$ & $\begin{array}{l}21.2(\mathrm{~s}) \\
\times \quad 1.52\end{array}$ & $\begin{array}{l}21.5(\mathrm{~s}) \\
\times 1.54\end{array}$ & - & - \\
\hline
\end{tabular}

Table A.4: Elastic stiffness matrix (2D): comparison of OptVS, OptV, OptV2, OptV1 and base codes in Matlab (top left), Octave (top right) and Python (bottom) giving time in seconds (top value) and OptVS speedup (bottom value). 


\section{B Proof of Lemma 5.1}

To prove Lemma 5.1 we introduce the following matrix $\mathbb{B}_{l}$ :

$$
\mathbb{B}_{l}=\left(\begin{array}{cc}
\delta_{l, 1} & 0 \\
0 & \delta_{l, 2} \\
\delta_{l, 2} & \delta_{l, 1}
\end{array}\right) \quad \text { if } d=2, \quad \text { and } \quad \mathbb{B}_{l}=\left(\begin{array}{ccc}
\delta_{l, 1} & 0 & 0 \\
0 & \delta_{l, 2} & 0 \\
0 & 0 & \delta_{l, 3} \\
\delta_{l, 2} & \delta_{l, 1} & 0 \\
0 & \delta_{l, 3} & \delta_{l, 2} \\
\delta_{l, 3} & 0 & \delta_{l, 1}
\end{array}\right) \quad \text { if } d=3
$$

Thus we have $\underline{\boldsymbol{\epsilon}}\left(\boldsymbol{\lambda}_{l, \alpha}\right)=\mathbb{B}_{l} \nabla \lambda_{\alpha}$ and then

$$
\underline{\epsilon}^{t}\left(\boldsymbol{\lambda}_{n, \beta}\right) \mathbb{C} \underline{\underline{\epsilon}}\left(\boldsymbol{\lambda}_{l, \alpha}\right)=\nabla \lambda_{\beta}^{t} \mathbb{B}_{n}^{t} \mathbb{C B}_{l} \nabla \lambda_{\alpha} .
$$

Moreover we have $\mathbb{C}=\lambda \mathbb{C}_{0}+\mu \mathbb{C}_{1}$ with

$$
\mathbb{C}_{0}=\left(\begin{array}{cc}
\mathbb{1}_{d} & \mathbb{O}_{d, 2 d-3} \\
\mathbb{O}_{2 d-3, d} & \mathbb{O}_{2 d-3}
\end{array}\right)_{3(d-1) \times 3(d-1)} \quad \text { and } \quad \mathbb{C}_{1}=\left(\begin{array}{cc}
2 \mathbb{I}_{d} & \mathbb{O}_{d, 2 d-3} \\
\mathbb{O}_{2 d-3, d} & \mathbb{I}_{2 d-3}
\end{array}\right)_{3(d-1) \times 3(d-1)}
$$

Thus we obtain

$$
\underline{\boldsymbol{\epsilon}}^{t}\left(\boldsymbol{\lambda}_{n, \beta}\right) \mathbb{C} \underline{\boldsymbol{\epsilon}}\left(\boldsymbol{\lambda}_{l, \alpha}\right)=\lambda \nabla \lambda_{\beta}^{t} \mathbb{B}_{n}^{t} \mathbb{C}_{0} \mathbb{B}_{l} \nabla \lambda_{\alpha}+\mu \nabla \lambda_{\beta}^{t} \mathbb{B}_{n}^{t} \mathbb{C}_{1} \mathbb{B}_{l} \nabla \lambda_{\alpha} .
$$

Denoting $\mathbb{Q}^{n, l}=\mathbb{B}_{n}^{t} \mathbb{C}_{0} \mathbb{B}_{l}$ and $\mathbb{S}^{n, l}=\mathbb{B}_{n}^{t} \mathbb{C}_{1} \mathbb{B}_{l}$ we obtain (5.6) which ends the proof of Lemma 5.1

\section{Remaining routines}

C.1 Gradients of the barycentric coordinates

Let $T_{k}$ be a $d$-simplex of $\mathbb{R}^{d}$ with vertices $\mathrm{q}^{0}, \ldots, \mathrm{q}^{d}$, and $\hat{T}$ be the reference $d$-simplex with vertices $\hat{\mathrm{q}}^{0}, \ldots, \hat{\mathrm{q}}^{d}$ where $\hat{\mathrm{q}}^{0}=\mathbf{0}_{d}$ and $\hat{\mathrm{q}}^{i}=\boldsymbol{e}_{i}, \forall i \in\{1, \ldots, d\}$.

Let $\mathcal{F}_{k}$ be the bijection from $\hat{T}$ to $T_{k}$ defined by q $=\mathcal{F}_{k}(\hat{\mathrm{q}})=\mathbb{B}_{k} \hat{\mathrm{q}}+\mathrm{q}^{0}$ where $\mathbb{B}_{k} \in$ $\mathcal{M}_{d}(\mathbb{R})$ is such that its $i$-th column is equal to $\mathrm{q}^{i}-\mathrm{q}^{0}$, for all $i \in\{1, \ldots, d\}$.

The barycentric coordinates of $\hat{\mathrm{q}}=\left(\hat{x}_{1}, \ldots, \hat{x}_{d}\right) \in \hat{T}$ are given by $\hat{\lambda}_{0}=1-\sum_{i=1}^{d} \hat{x}_{i}$, and $\hat{\lambda}_{i}=\hat{x}_{i}, \forall i \in\{1, \ldots, d\}$. The barycentric coordinates of $\mathrm{q}=\left(x_{1}, \ldots, x_{d}\right) \in T_{k}$ are given by $\lambda_{k, i}(\mathrm{q})=\hat{\lambda}_{i} \circ \mathcal{F}_{k}^{-1}(\mathrm{q})$ and we have

$$
\nabla \lambda_{k, i}(\mathrm{q})=\mathbb{B}_{k}^{-t} \hat{\nabla} \hat{\lambda}_{i}(\hat{\mathrm{q}}), \forall i \in\{0, \ldots, d\},
$$

with $\hat{\nabla} \hat{\lambda}_{0}(\hat{\mathrm{q}})=\left(\begin{array}{c}-1 \\ \ldots \\ -1\end{array}\right), \hat{\nabla} \hat{\lambda}_{i}=\boldsymbol{e}_{i}, \forall i \in\{1, \ldots, d\}$. Note that gradients are constant. Let

$$
\hat{\mathbb{G}}=\left(\hat{\nabla} \hat{\lambda}_{0}, \ldots, \hat{\nabla} \hat{\lambda}_{d}\right)=\left(\begin{array}{ccccc}
-1 & 1 & 0 & \ldots & 0 \\
-1 & 0 & 1 & \ddots & \vdots \\
\vdots & \vdots & \ddots & \ddots & 0 \\
-1 & 0 & \ldots & 0 & 1
\end{array}\right) .
$$

Then computing the gradients of the barycentric coordinates is equivalent to solve $(d+1)$ linear systems, written in matrix form as follows:

$$
\mathbb{B}_{k}^{t} \mathbb{G}_{k}=\hat{\mathbb{G}},
$$


where $\mathbb{G}_{k}=\left(\nabla \lambda_{k, 0}(\mathrm{q}), \ldots, \nabla \lambda_{k, d}(\mathrm{q})\right) \in \mathcal{M}_{d, d+1}(\mathbb{R})$.

For each $d$-simplex one has to calculate $(d+1)$ gradients and thus to determine $(d+1) \mathrm{n}_{\mathrm{me}}$ vectors of dimension $d$.

The vectorization of the calculation of the gradients is done by rewriting the equations (C.2), for $k=1, \ldots, \mathrm{n}_{\text {me }}$, under an equivalent form of a large block diagonal sparse system of size $N=d \times \mathrm{n}_{\text {me }}$, with $d$-by- $d$ diagonal blocks given by:

$$
\left(\begin{array}{cccc}
\mathbb{B}_{1}^{t} & \mathbb{O} & \ldots & \mathbb{O} \\
\mathbb{O} & \ddots & \ddots & \vdots \\
\vdots & \ddots & \ddots & \mathbb{O} \\
\mathbb{O} & \ldots & \mathbb{O} & \mathbb{B}_{\mathrm{n}_{\mathrm{me}}}^{t}
\end{array}\right)_{N \times N}\left(\begin{array}{c}
\mathbb{G}_{1} \\
\mathbb{G}_{2} \\
\vdots \\
\mathbb{G}_{\mathrm{n}_{\mathrm{me}}}
\end{array}\right)_{N \times(d+1)}=\left(\begin{array}{c}
\hat{\mathbb{G}} \\
\hat{\mathbb{G}} \\
\vdots \\
\hat{\mathbb{G}}
\end{array}\right)_{N \times(d+1)}
$$

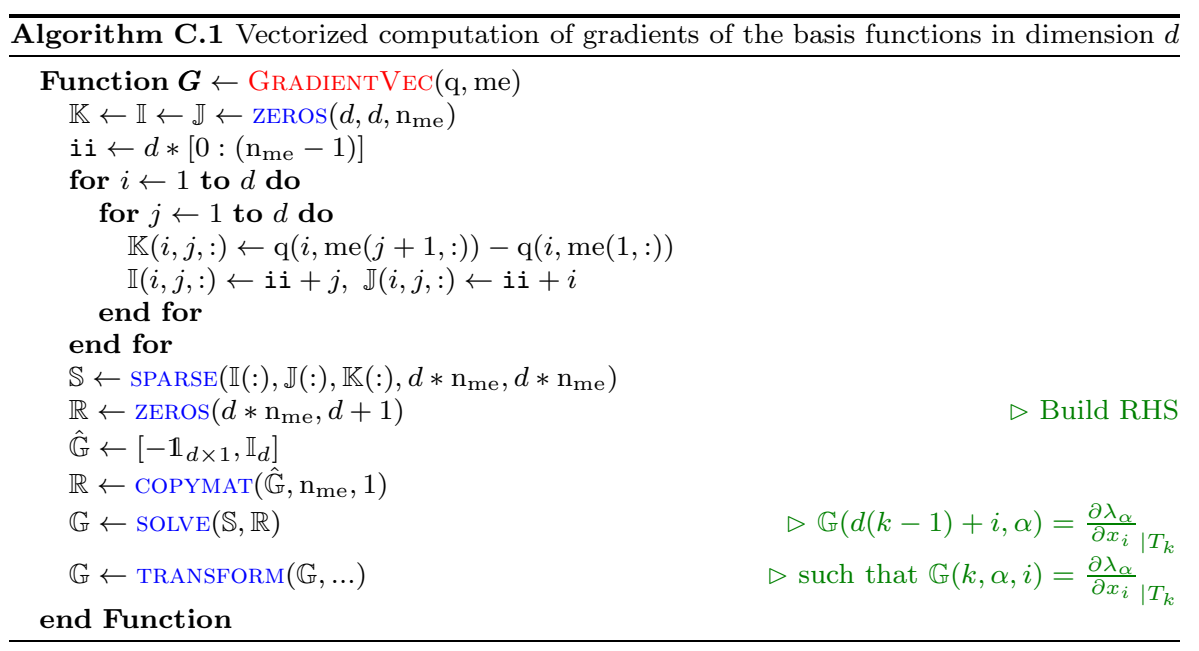

The performance of this algorithm may be improved by writing specific algorithms in each dimension $d=1,2$ or 3 (see Appendix A in [6]).

C.2 Elastic stiffness matrix assembly : algorithm using the symmetry

When the assembly matrix is symmetric, one may improve the performance of Algorithm 5.3 by using the symmetry of the element matrices (see Section 4), which leads to the following algorithm: 


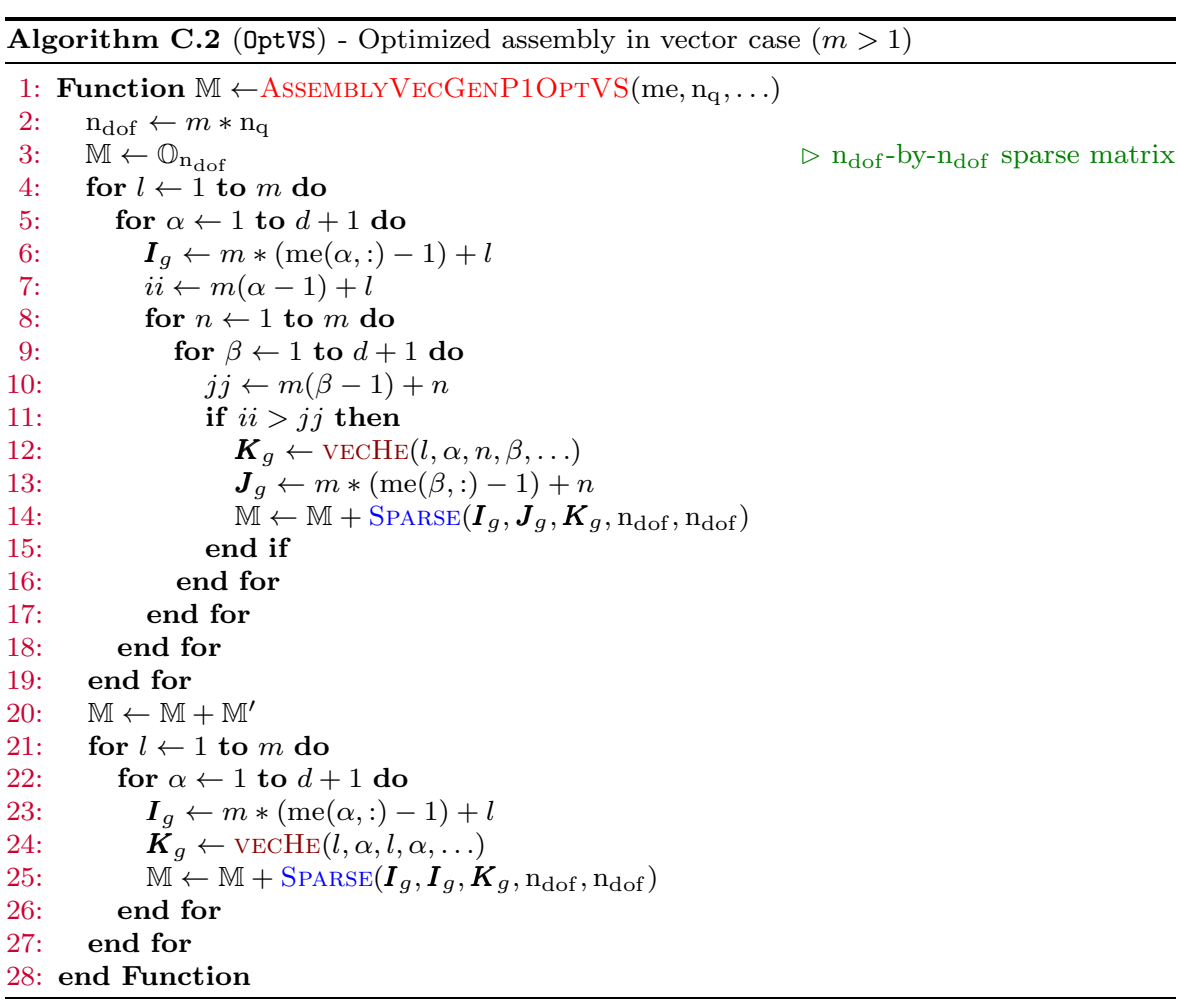

\section{Extension to $\mathbb{P}_{k}$-Lagrange finite elements}

In this section we adapt the optimized algorithm of Section 4 to the case of finite elements of higher order. For simplicity, we consider the assembly algorithm on the example of the mass matrix.

The mesh used is adapted to $\mathbb{P}_{k}$ finite elements and is called a "P्P $k$-mesh". Only arrays $\mathrm{q}$ and me differ between the usual mesh and the $\mathbb{P}_{k}$-mesh. In the $\mathbb{P}_{k}$-mesh, q contains the coordinates of the nodal points associated to the $\mathbb{P}_{k}$ finite elements and me is of dimension $\mathrm{n}_{\mathrm{dfe}}$-by- $\mathrm{n}_{\text {me }}$, where $\mathrm{n}_{\mathrm{dfe}}$ is the local number of $\mathbb{P}_{k}$-nodes in a $d$-simplex $K: \mathrm{n}_{\mathrm{dfe}}=\frac{(d+k) !}{d ! k !}$, as shown in the table below.

\begin{tabular}{lccl}
\hline name & type & dimension & description \\
\hline $\mathrm{n}_{\mathrm{dfe}}$ & integer & 1 & local number of $\mathbb{P}_{k}$-nodes in a $d$-simplex \\
$\mathrm{n}_{\mathrm{q}}$ & integer & 1 & number of $\mathbb{P}_{k}$-nodes \\
$\mathrm{q}$ & double & $\mathrm{d} \times \mathrm{n}_{\mathrm{q}}$ & array of $\mathbb{P}_{k}$-node coordinates \\
$\mathrm{me}$ & integer & $\mathrm{n}_{\mathrm{dfe}} \times \mathrm{n}_{\mathrm{me}}$ & $\left(\mathbb{P}_{k}\right)$ connectivity array \\
\hline
\end{tabular}

By construction, the total number of degrees of freedom of a $\mathbb{P}_{k}$-mesh is its number of nodal points. One may use for example gmsh [16] to generate a $\mathbb{P}_{k}$-mesh in $2 \mathrm{D}$ or in $3 \mathrm{D}$.

First, we need to introduce some notations: let $\mathcal{S}_{d}^{k}$ be the set of multi-indices given by

$$
\mathcal{S}_{d}^{k}=\left\{\boldsymbol{\alpha}=\left(\alpha_{1}, \ldots, \alpha_{d+1}\right) \in \mathbb{N}^{d+1} \text { such that }|\boldsymbol{\alpha}|:=\sum_{i=1}^{d+1} \alpha_{i}=k\right\}
$$


with $\# \mathcal{S}_{d}=N$. Then the $\mathbb{P}_{k}$ basis functions $\varphi_{\boldsymbol{\alpha}}$ on a $d$-simplex $K$ may be deduced from the barycentric coordinates $\left\{\lambda_{j}\right\}_{j=1}^{d+1}$

$$
\varphi_{\boldsymbol{\alpha}}=\prod_{l=1}^{d+1} \prod_{j=0}^{\alpha_{l}-1} \frac{k \lambda_{l}-j}{j+1}, \quad \forall \boldsymbol{\alpha} \in \mathcal{S}_{d}^{k}
$$

or equivalently, noticing that $\varphi_{\boldsymbol{\alpha}}$ is a polynomial in the variable $\left(\lambda_{1}, \ldots, \lambda_{d+1}\right)$ and introducing a multi-index $\boldsymbol{\mu}=\left(\mu_{1}, \ldots, \mu_{d+1}\right) \in \mathbb{N}^{d+1}$, we have

$$
\varphi_{\boldsymbol{\alpha}}=\sum_{|\boldsymbol{\mu}| \leq k} a_{\boldsymbol{\mu}}(\boldsymbol{\alpha})\left(\prod_{j=1}^{d+1} \lambda_{j}^{\mu_{j}}\right)
$$

All the non-zero $a_{\boldsymbol{\mu}}(\boldsymbol{\alpha})$ values can be computed from D.2 and depend only on $\boldsymbol{\alpha}, d$ and $k$.

As in the previous sections, the assembly algorithm of the mass matrix is based on the vectorization of the local mass matrix $\mathbb{M}^{e}$ on $K$, which is an $N$-by- $N$ matrix given by

$$
\mathbb{M}_{I(\boldsymbol{\alpha}), I(\boldsymbol{\beta})}^{e}(K)=\int_{K} \varphi_{\boldsymbol{\alpha}} \varphi_{\boldsymbol{\beta}} d \mathrm{q}, \quad \forall(\boldsymbol{\alpha}, \boldsymbol{\beta}) \in \mathcal{S}_{d}^{k} \times \mathcal{S}_{d}^{k}
$$

where $I: \mathcal{S}_{d}^{k} \longrightarrow\{1, \ldots, N\}$ is the local numbering choice.

We then introduce a formula of the same type as (4.3) to vectorize the computation of $\mathbb{M}^{e}$. Using (D.3), we have for all $(\boldsymbol{\alpha}, \boldsymbol{\beta}) \in \mathcal{S}_{d}^{k} \times \mathcal{S}_{d}^{k}$

$$
\int_{K} \varphi_{\boldsymbol{\alpha}} \varphi_{\boldsymbol{\beta}} d \mathrm{q}=\sum_{|\boldsymbol{\mu}| \leq k} \sum_{|\boldsymbol{\nu}| \leq k} a_{\boldsymbol{\mu}}(\boldsymbol{\alpha}) a_{\boldsymbol{\nu}}(\boldsymbol{\beta}) \int_{K} \prod_{j=1}^{d+1} \lambda_{j}^{\mu_{j}+\nu_{j}} d \mathrm{q} .
$$

Then, using formula 2.4 we obtain

$$
\int_{K} \varphi_{\boldsymbol{\alpha}} \varphi_{\boldsymbol{\beta}} d \mathrm{q}=d !|K| C_{\boldsymbol{\alpha}, \boldsymbol{\beta}}
$$

where the constant $C_{\boldsymbol{\alpha}, \boldsymbol{\beta}}$ does not depend on $K$ and is given by

$$
C_{\boldsymbol{\alpha}, \boldsymbol{\beta}}=\sum_{|\boldsymbol{\mu}| \leq k} \sum_{|\boldsymbol{\nu}| \leq k} a_{\boldsymbol{\mu}}(\boldsymbol{\alpha}) a_{\boldsymbol{\nu}}(\boldsymbol{\beta}) \frac{\prod_{i=1}^{d+1}\left(\mu_{i}+\nu_{i}\right) !}{(d+|\boldsymbol{\mu}|+|\boldsymbol{\nu}|) !} .
$$

Using (D.4), we can now extend Algorithm 4.4 (with $w=1$ ) to the $\mathbb{P}_{k}$ finite element case. This leads to the vectorized algorithm of the mass matrix given in Algorithm D.1

Remark D.1 We have considered the extension of the OptV2 algorithm to finite elements of higher order. The main idea is that all the steps of Section 4 remain valid for $\mathbb{P}_{k}$ finite elements, if one replaces $(d+1)$ by $\mathrm{n}_{\mathrm{dfe}}$, and with $\mathrm{q}$ and me defined above. Then, one may derive from Algorithm D.1 the other optimized versions OptV and OptVS for the $\mathbb{P}_{k}$ case, as in Section 4. 


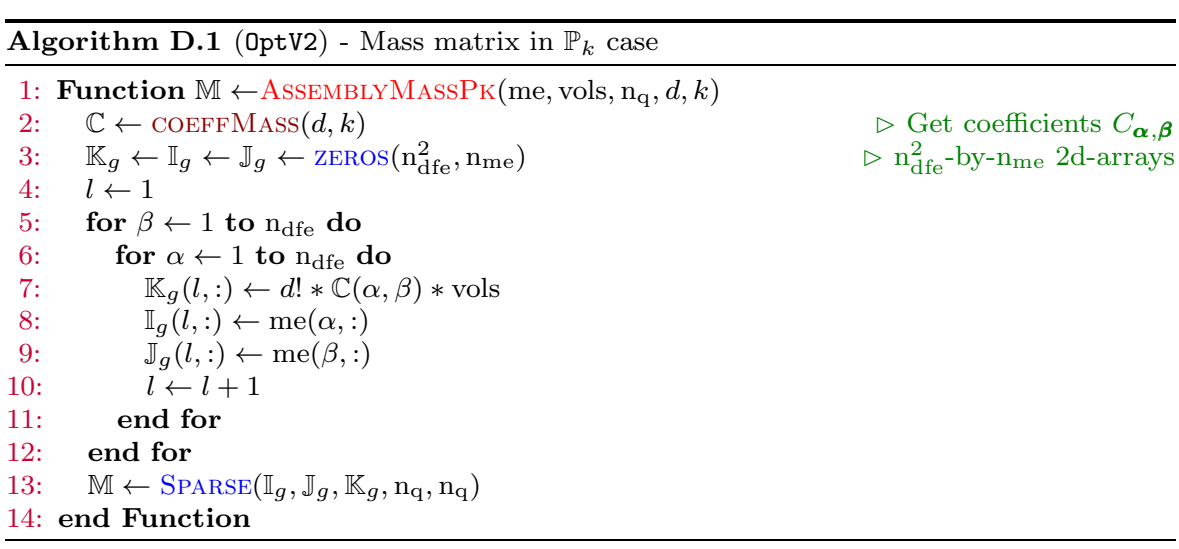

In Table D.1 using Matlab, we show the computation times versus the number of $\mathbb{P}_{k}$ nodes, for Algorithm 4.4 (with $w=1$ ), and for Algorithm D.1 with $k=1,2,3,4,5,6$. We observe that the computation times are almost the same for Algorithm 4.4 and AlgorithmD.1 with $k=1$. Moreover, for a fixed number of nodes, the computation times increase slowly with the degree of the polynomials: for a million of nodes, the computation time with $\mathbb{P}_{5}$ finite elements is twice the one for $\mathbb{P}_{1}$ finite elements.

\begin{tabular}{|r||c|c|c|c|c|c|c|}
\hline $\mathrm{n}_{\text {dof }}$ & $\mathrm{P} 1 \mathrm{OptV} 2$ & $\mathrm{Pk}(\mathrm{k}=1)$ & $\mathrm{Pk}(\mathrm{k}=2)$ & $\mathrm{Pk}(\mathrm{k}=3)$ & $\mathrm{Pk}(\mathrm{k}=4)$ & $\mathrm{Pk}(\mathrm{k}=5)$ & $\mathrm{Pk}(\mathrm{k}=6)$ \\
\hline \hline $3.10^{4}$ & 0.535 & 0.543 & 0.459 & 0.544 & 0.707 & 0.982 & 1.350 \\
\hline $1.210^{5}$ & 2.322 & 2.500 & 2.025 & 2.407 & 3.184 & 4.389 & 5.696 \\
\hline $5.10^{5}$ & 10.885 & 13.203 & 9.811 & 11.684 & 15.184 & 19.766 & 25.340 \\
\hline $10^{6}$ & 22.744 & 28.362 & 22.635 & 25.656 & 33.314 & 42.812 & 54.782 \\
\hline
\end{tabular}

Table D.1: 3D Mass matrix : computational cost versus $\mathrm{n}_{\text {dof }}$, with Matlab, for OptV2 code : Algorithm 4.4 with $w=1$ ) (column 1), and with Algorithm D.1 for $k=1,2,3,4,5,6$ (columns 2 to 7 ).

Acknowledgements The authors would like to thank Prof. H-P. Langtangen for his many constructive comments that led to a better presentation of the paper.

\section{References}

1. I. Anjam and J. Valdman. Fast MATLAB assembly of FEM matrices in 2D and 3D: Edge elements. arXiv:1409.4618v1, 2014.

2. L. Chen. Programming of Finite Element Methods in Matlab. http://math.uci.edu/ chenlong/226/Ch3FEMCode.pdf 2011.

3. L. Chen. iFEM, a Matlab software package. http://math.uci.edu/ chenlong/programming.html 2013.

4. Z. Chen. Finite Element Methods and their Applications. Springer, scientific computation edition, 2005.

5. P. G. Ciarlet. The Finite Element Method for Elliptic Problems. SIAM, Philadelphia, 2002 
6. F. Cuvelier, C. Japhet, and G. Scarella. An efficient way to perform the assembly of finite element matrices in vector languages. http://hal.archives-ouvertes.fr/hal-00931066 2014.

7. F. Cuvelier, C. Japhet, and G. Scarella. OptFEM packages. http://www.math.univ-paris13.fr/ cuvelier/software 2015.

8. M. Dabrowski, M. Krotkiewski, and D. W. Schmid. Milamin: Matlab-based finite element method solver for large problems. Geochem. Geophys. Geosyst., 9, 2008.

9. T. A. Davis. Direct Methods for Sparse Linear Systems. Society for Industrial and Applied Mathematics, 2006.

10. T. A. Davis. SuiteSparse packages, released 4.2.1. http://faculty.cse.tamu.edu/davis/suitesparse.html, 2013.

11. G. Dhatt, E. Lefrançois, and G. Touzot. Finite Element Method. Wiley, 2012.

12. Scilab Enterprises. Scilab. http://www.scilab.org/ 2015.

13. Python Software Foundation. Python. http://www.python.org/, 2013.

14. R Foundation. The R Project for Statistical Computing. http://www.r-project.org/ 2015.

15. S. Funken, D. Praetorius, and P. Wissgott. Efficient implementation of adaptive P1FEM in MATLAB. Computational Methods in Applied Mathematics, 11 (4):460-490, 2011.

16. C. Geuzaine and J.-F. Remacle. Gmsh: a three-dimensional finite element mesh generator with built-in pre- and post-processing facilities. International Journal for Numerical Methods in Engineering, 79(11):1309-1331, 2009.

17. A. Hannukainen and M. Juntunen. Implementing the Finite Element Assembly in Interpreted Languages, 2012. Preprint, Aalto University.

18. F. Hecht. New development in freefem++. J. Numer. Math., 20 (3-4):251-265, 2012.

19. F. Hecht, O. Pironneau, J. Morice, A. Le Hyaric, and K. Ohtsuka. Freefem++. http://www. freefem.org/ff++

20. J. S. Hesthaven and T. Warburton. Nodal Discontinuous Galerkin Methods, Algorithms, Analysis, and Applications, volume 54. Springer, texts in Applied Mathematics edition, 2008.

21. C. Johnson. Numerical Solution of Partial Differential Equations by the Finite Element Method. Dover Publications, Inc, 2009.

22. Julia. http://julialang.org/, 2015.

23. J. Koko. Vectorized Matlab codes for linear two-dimensional elasticity. Scientific Programming, 15(3):157-172, 2007.

24. H. P. Langtangen and X. Cai. On the efficiency of Python for high-performance computing: A case study involving stencil updates for partial differential equations. In Modeling, Simulation and Optimization of Complex Processes, pages 337-358. Springer, 2008.

25. A. Logg, K.-A. Mardal, and G. N. Wells et al. Matlab Implementation of the Finite Element Method in Elasticity. Automated Solution of Differential Equations by the Finite Element Method, 2012.

26. B. Lucquin and O. Pironneau. Introduction to Scientific Computing. John Wiley \& Sons Ltd, 1998.

27. Mathworks. Matlab. http://www.mathworks.com 2014.

28. NVIDIA. Cusp, a C++ Templated Library for sparse linear algebra on CUDA. https://developer.nvidia.com/cusp, 2013.

29. NVIDIA. Thrust, a C++ template library for CUDA based on the Standard Template Library (STL). https://developer.nvidia.com/thrust 2013.

30. Octave community. GNU Octave 3.8.1, 2014.

31. A. Quarteroni. Numerical Models for Differential Problems. Springer, 2014.

32. A. Quarteroni, F. Saleri, and P. Gervasio. Scientific Computing with MATLAB and Octave. Springer, texts in Computational Science and Engineering edition, 2013.

33. A. Quarteroni and A. Valli. Numerical Approximation of Partial Differential Equations. Springer, 2008.

34. T. Rahman and J. Valdman. Fast MATLAB assembly of FEM matrices in 2D and 3D: Nodal elements. Appl. Math. Comput., 219 (13):7151-7158, 2013.

35. V. Thomée. Galerkin Finite Element Method for Parabolic Problems. Springer, 1994. 